This item was submitted to Loughborough's Research Repository by the author.

Items in Figshare are protected by copyright, with all rights reserved, unless otherwise indicated.

\title{
Confluences of the Painlevé equations, Cherednik algebras and q-Askey scheme
}

PLEASE CITE THE PUBLISHED VERSION

http://dx.doi.org/10.1088/0951-7715/29/9/2565

PUBLISHER

(C) IOP Publishing Ltd \& London Mathematical Society

VERSION

AM (Accepted Manuscript)

LICENCE

CC BY-NC-ND 4.0

REPOSITORY RECORD

Mazzocco, Marta. 2016. "Confluences of the Painlevé Equations, Cherednik Algebras and Q-askey Scheme". Loughborough University. https://hdl.handle.net/2134/21151. 


\title{
CONFLUENCES OF THE PAINLEVÉ EQUATIONS, CHEREDNIK ALGEBRAS AND Q-ASKEY SCHEME.
}

\author{
MARTA MAZZOCCO ${ }^{\dagger}$
}

\begin{abstract}
In this paper we produce seven new algebras as confluences of the Cherednik algebra of type $\check{C}_{1} C_{1}$ and we characterise their spherical-subalgebras.

The limit of the spherical sub-algebra of the Cherednik algebra of type $\check{C}_{1} C_{1}$ is the monodromy manifold of the Painlevé VI equation [35]. Here we prove that by considering the limits of the spherical sub-algebras of our new confluent algebras, one obtains the monodromy manifolds of all other Painlevé differential equations. Moreover, we introduce confluent versions of the Zhedanov algebra and prove that each of them (quotiented by their Casimir) is isomorphic to the corresponding spherical sub-algebra of our new confluent Cherednik algebras. We show that in the basic representation our confluent Zhedanov algebras act as symmetries of certain elements of the q-Askey scheme, thus setting a stepping stone towards the solution of the open problem of finding the corresponding quantum algebra for each element of the q-Askey scheme.

These results establish a new link between the theory of the Painlevé equations and the theory of the q-Askey scheme making a step towards the construction of a representation theoretic approach for the Painlevé theory.
\end{abstract}

\section{Contents}

1. Introduction

2. Notation and background on the Cherednik algebra of type $\check{C}_{1} C_{1}$

2.1. Automorphisms of the Cherednik algebra of type $\check{C}_{1} C_{1}$

2.2. Zhedanov algebra and Askey Wilson polynomials

2.3. An important cubic relation and the spherical sub-algebra $e \mathcal{H} e$

3. Derivation and first properties of the confluent Cherednik algebras

3.1. Derivation of $\mathcal{H}_{V}$

3.2. Derivation of $\mathcal{H}_{I V}, \mathcal{H}_{I I}, \mathcal{H}_{I I}, \mathcal{H}_{I}$

3.3. Derivation of $\mathcal{H}_{I I I^{D_{7}}}$, and $\mathcal{H}_{I I I^{D_{8}}}$

4. Confluent spherical sub-algebras and Painlevé cubics 14

4.1. Spherical sub-algebra of $\mathcal{H}_{V}$ and PV monodromy manifold 15

4.2. Spherical sub-algebra of $\mathcal{H}_{I V}$ and PIV monodromy manifold 17

4.3. Spherical sub-algebra of $\mathcal{H}_{I I I}$ and PIII monodromy manifold 18

4.4. Spherical sub-algebra of $\mathcal{H}_{I I I^{D_{7}}}$ and $P I I I^{D_{7}}$ monodromy manifold 19

4.5. Spherical sub-algebra of $\mathcal{H}_{I I I^{D_{8}}}$ and $P I I I^{D_{8}}$ monodromy manifold 20

4.6. Spherical sub-algebra of $\mathcal{H}_{I I}$ and PII monodromy manifold 21

4.7. Spherical sub-algebra of $\mathcal{H}_{I}$ and PI monodromy manifold 21

5. $\quad$ Embedding of the (confluent) Cherednik algebras into $\operatorname{Mat}\left(2, \mathbb{T}_{q}\right) \quad 22$

\footnotetext{
${ }^{\dagger}$ Department of Mathematical Sciences, Loughborough University, UK. Email: m.mazzocco@lboro.ac.uk, Phone: +44 1509 223187, Fax: +44 (0)1509 223969.
} 
6. Confluent Zhedanov algebras and q-Askey scheme 27

6.1. Representation of $\mathcal{Z}_{V}$ and continuous dual q-Hahn polynomials $\quad 29$

6.2. Big $q$-Jacobi polynomials 30

6.3. Representation of $\mathcal{Z}_{I V}$ and Big $q$-Laguerre Polynomials 31

6.4. Representation of $\mathcal{Z}_{I I I}$ and Al-Salam-Chihara Polynomials 32

6.5. Representation of $\mathcal{Z}_{I I I}^{D_{7}}$ and continuous Big $q$-Hermite Polynomials 33

6.6. Representation of $\mathcal{Z}_{I I I}^{D_{8}}$ and continuous $q$-Hermite Polynomials 33

6.7. Representation of $\mathcal{Z}_{I I}$ and little $q$-Laguerre/Wall polynomials 34

6.8. Representation of $\mathcal{Z}_{I}$ and a special case of the little $q$-Laguerre/Wall polynomials 34

Appendix A The Painlevé monodromy manifolds 35

$\begin{array}{ll}\text { References } & 36\end{array}$

\section{Introduction}

The relationship between the theory of the Painlevé equations and special or orthogonal polynomials is a very famous one and could be resumed by saying that thanks to the $\tau$-function structure of the Painlevé equations, some of their special solutions are related to special or orthogonal polynomials either directly, i.e. some rational solutions of the Painlevé equations are ratios of special polynomials $[50,49$, $36,37,38,32,34,51,47,9]$, or indirectly, i.e. some random matrix integrals which can be expressed by classical orthogonal polynomials have Fredholm determinants which can be expressed in terms of special solutions of the Painlevé equations $[46,3,14,4]$.

It this paper we present a new relation between the theory of the Painlevé equations and q-polynomials belonging to the q-Askey scheme [23]. This link does not rely on the $\tau$-function structure nor on choosing special solutions, it is indeed a much deeper and more conceptual relation that has allowed the author to discover seven new confluent Cherednik algebras and to prove several interesting results about them.

Let us start from the Painlevé sixth equation [15, 42, 16] which describes the monodromy preserving deformations of a rank 2 Fuchsian system with four simple poles $a_{1}, a_{2}, a_{3}$ and $\infty$. The solution of this Fuchsian system is in general a multivalued analytic vector-function in the punctured Riemann sphere $\mathbb{P}^{1} \backslash\left\{a_{1}, a_{2}, a_{3}, \infty\right\}$ and its multivaluedness is described by the so-called monodromy group, i.e. a subgroup of $S L_{2}(\mathbb{C})$ generated by the images $M_{1}, M_{2}, M_{3}$ of the generators of the fundamental group under the anti-homomorphism:

$$
\rho: \pi_{1}\left(\mathbb{P}^{1} \backslash\left\{a_{1}, a_{2}, a_{3}, \infty\right\}, \lambda_{0}\right) \rightarrow S L_{2}(\mathbb{C}) .
$$

The moduli space $\mathcal{M} / \Gamma$ of monodromy representations $\rho$ up to Jordan equivalence, with prescribed local monodromy (i.e. prescribed conjugacy class for each $\left.M_{1}, M_{2}, M_{3}\right)$, is realised as an affine cubic surface [20] which coincides with the center of the Cherednik algebra of type $\check{C}_{1} C_{1}$ for $q=1[35,11]$. In [5] this affine cubic surface was explicitly quantised leading to the Zhedanov algebra, which is to be isomorphic [24] to the spherical subalgebra of the Cherednik algebra of type $\check{C}_{1} C_{1}$ i.e. the algebra $\mathcal{H}$ generated by four elements $T_{0}, T_{1}, X, W$ with the following 
relations ${ }^{1}[7,39,33,43]$ :

$$
\begin{aligned}
X W=W X & =1, \\
\left(T_{1}+a b\right)\left(T_{1}+1\right) & =0, \\
\left(T_{0}+q^{-1} c d\right)\left(T_{0}+1\right) & =0, \\
\left(T_{1} X+a\right)\left(T_{1} X+b\right) & =0, \\
\left(q T_{0} W+c\right)\left(q T_{0} W+d\right) & =0,
\end{aligned}
$$

where $a, b, c, d, q \in \mathbb{C}^{\star}$.

In this paper, we produce seven new algebras as confluences of the Cherednik algebra of type $\check{C}_{1} C_{1}$ in such a way that their spherical-sub-algebras tend in the limit to the monodromy manifolds of all other Painlevé differential equations:

Definition 1.1. Let $a, b, c, q \in \mathbb{C}^{\star}$. The confluent Cherednik algebras $\mathcal{H}_{V}, \mathcal{H}_{I V}$, $\mathcal{H}_{I I I}, \mathcal{H}_{I I I^{D_{7}}}, \mathcal{H}_{I I I^{D_{8}}}, \mathcal{H}_{I I}$ are the unital associative $\mathbb{C}$-algebras generated by four elements $X, W, T_{0}, T_{1}$ satisfying the following relations respectively:

- $\mathcal{H}_{V}$ :

$$
\begin{array}{r}
X W=W X=1, \\
\left(T_{1}+a b\right)\left(T_{1}+1\right)=0, \\
T_{0}\left(T_{0}+1\right)=0, \\
\left(T_{1} X+a\right)\left(T_{1} X+b\right)=0, \\
q T_{0} W+c=X\left(T_{0}+1\right),
\end{array}
$$

- $\mathcal{H}_{I V}$ :

- $\mathcal{H}_{I I I}$ :

$$
\begin{array}{r}
X W=W X=0, \\
\left(T_{1}-b^{2}\right)\left(T_{1}+1\right)=0, \\
T_{0}\left(T_{0}+1\right)=0, \\
T_{1} X+b=W\left(T_{1}-b^{2}+1\right), \\
q T_{0} W+c=X\left(T_{0}+1\right),
\end{array}
$$

\section{- $\mathcal{H}_{I I I^{D_{7}}}$ :}

$$
\begin{array}{r}
X W=W X=1, \\
\left(T_{1}+a b\right)\left(T_{1}+1\right)=0, \\
T_{0}^{2}=0 \\
\left(T_{1} X+a\right)\left(T_{1} X+b\right)=0 \\
q T_{0} W-\sqrt{q}=X T_{0} .
\end{array}
$$

\footnotetext{
${ }^{1}$ Note that here $W=X^{-1}$. However we prefer here to use the notation $W$ as in the confluent cases the generator $X$ may become singular.
} 
- $\mathcal{H}_{I I I^{D_{8}}}$ :

$$
\text { - } \mathcal{H}_{I I} \text { : }
$$

$$
\begin{array}{r}
X W=W X=1, \\
T_{1}\left(T_{1}+1\right)=0, \\
T_{0}^{2}=0, \\
T_{1} X-W\left(T_{1}+1\right)=0, \\
q T_{0} W-\sqrt{q}=X T_{0} .
\end{array}
$$

It is worth noting that in the above definition, we have three different algebras corresponding to three different types of the third Painlevé equation [41].

The next set of results regards equivalent presentations for these confluent algebras. For the Cherednik algebra of type $\check{C}_{1} C_{1}$ the following result is well known [33]:

Lemma 1.2. $[33,35]$ The Cherednik algebra of type $\check{C}_{1} C_{1}$ is isomorphic as algebra to the unital associative $\mathbb{C}$-algebra with generators $V_{0}, \check{V}_{0}, V_{1}, \check{V}_{1}$ and relations:

$$
\begin{array}{r}
X W=W X=0, \\
\left(T_{1}-b^{2}\right)\left(T_{1}+1\right)=0, \\
T_{0}\left(T_{0}+1\right)=0, \\
T_{1} X=W\left(T_{1}-b^{2}+1\right), \\
q T_{0} W-\sqrt{q}=X\left(T_{0}+1\right) .
\end{array}
$$

where $k_{0}, k_{1}, u_{0}, u_{1}, q \in \mathbb{C}^{\star}$. The isomorphism is explicitly given by

$$
\phi\left(T_{0}\right)=k_{0} V_{0}, \quad \phi\left(T_{1}\right)=u_{1} \check{V}_{1}, \quad \phi(X)=q^{1 / 2} V_{0} \check{V}_{0}, \quad \phi(W)=\check{V}_{1} V_{1},
$$

and for the parameters

$$
a=-\frac{u_{1}}{k_{1}}, \quad b=k_{1} u_{1}, \quad c=-q^{\frac{1}{2}} \frac{k_{0}}{u_{0}}, \quad d=q^{1 / 2} u_{0} k_{0} .
$$

In Theorem 3.2 we prove that all the confluent algebras $\mathcal{H}_{V}, \ldots, \mathcal{H}_{I I}$ are also isomorphic as algebras to some unital associative $\mathbb{C}$-algebras with generators $V_{0}$, $\check{V}_{0}, V_{1}, \check{V}_{1}$ and confluent analogues of the relations $(1.36,1.37,1.38,1.39,1.40)$. This allows to produce one further confluent algebra that was not visible in the previous presentation:

Definition 1.3. Let $q \in \mathbb{C}^{\star}$. The confluent Cherednik algebra $\mathcal{H}_{I}$ is the algebra with generators $V_{0}, \check{V}_{0}, V_{1}, \check{V}_{1}$ and relations:

$$
\begin{aligned}
V_{0}\left(V_{0}+1\right) & =0 \\
V_{1}^{2} & =0 \\
\check{V}_{0}\left(\check{V}_{0}+1\right) & =0 \\
\check{V}_{1}\left(\check{V}_{1}+1\right) & =0
\end{aligned}
$$




$$
\begin{array}{r}
q^{1 / 2} \check{V}_{1} V_{1} V_{0}=\check{V}_{0}+1, \\
V_{0} \check{V}_{0}=0,
\end{array}
$$

Next, we deal with the spherical sub-algebras of each confluent Cherednik algebra. We start by selecting a symmetriser $e$ and three special elements denoted by $X_{1}, X_{2}, X_{3}$ such that $\left[e, X_{i}\right]=0, i=1,2,3$, and such that $\hat{X}_{i}:=e X_{i}, i=1,2,3$, generate the spherical sub-algebras of each confluent Cherednik algebra. We prove that such elements $X_{1}, X_{2}, X_{3}$ satisfy a cubic relation (see Propositions 4.1, 4.4, 4.7, $4.11,4.15,4.18,4.21$ ) and that in the limit such cubic relations coincide with the monodromy manifolds of the corresponding Painlevé equations as defined in [40, 48] (see Corollaries 4.2, 4.5, 4.8, 4.12, 4.16, 4.19, 4.22). In other words, one could say that the confluent Cherednik algebras introduced in this paper are such that their spherical sub-algebras produce a quantisation of the monodromy manifolds of the respective Painlevé equations.

In order to link the spherical sub-algebras $e \mathcal{H}_{V} e, \ldots, e \mathcal{H}_{I} e$ to the q-Askey scheme, we first need to introduce the confluent versions of the Zhedanov algebra $\mathcal{Z}$ given in Definition 2.2 in Subsection 2.2 here below [52]:

Definition 1.4. The confluent Zhedanov algebras $\mathcal{Z}_{V}, \mathcal{Z}_{I V}, \mathcal{Z}_{I I I}, \mathcal{Z}_{I I I}^{D_{7}}, \mathcal{Z}_{I I I}^{D_{8}}, \mathcal{Z}_{I I}$, $\mathcal{Z}_{I}$ are the algebras generated by three elements $K_{0}, K_{1}$ and $K_{2}$ which satisfy the following relations:

$$
\begin{array}{r}
q^{\frac{1}{2}} K_{0} K_{1}-q^{-\frac{1}{2}} K_{1} K_{0}=K_{2}, \\
q^{\frac{1}{2}} K_{1} K_{2}-q^{-\frac{1}{2}} K_{2} K_{1}=B K_{1}+C_{0} K_{0}+D_{0}, \\
q^{\frac{1}{2}} K_{2} K_{0}-q^{-\frac{1}{2}} K_{0} K_{2}=B K_{0}+D_{1},
\end{array}
$$

where the parameters $B, C_{0}, D_{0}, D_{1}$ are arbitrary non zero constants, or have a specific form according to the following table:

$$
\begin{aligned}
& B=\left\{\begin{array}{cc}
\text { arbitrary } & \text { for } \mathcal{Z}_{V}, \mathcal{Z}_{I V}, \mathcal{Z}_{I I I}, \mathcal{Z}_{I I}, \\
-\frac{(q-1)^{2}}{q} & \text { for } \mathcal{Z}_{I I I}^{D_{7}}, \mathcal{Z}_{I I I}^{D_{8}}, \\
-\frac{(q-1)^{2}}{\sqrt{q}} & \text { for } \mathcal{Z}_{I},
\end{array}\right. \\
& C_{0}=\left\{\begin{array}{cc}
\left(q-\frac{1}{q}\right)^{2}, & \text { for } \mathcal{Z}_{V}, \mathcal{Z}_{I I I}, \mathcal{Z}_{I I I}^{D_{7}}, \mathcal{Z}_{I I I}^{D_{8}}, \\
0, & \text { for } \mathcal{Z}_{I V}, \mathcal{Z}_{I I}, \mathcal{Z}_{I}
\end{array}\right. \\
& D_{0}=\left\{\begin{array}{cc}
\text { arbitrary } & \text { for } \mathcal{Z}_{V}, \mathcal{Z}_{I V}, \mathcal{Z}_{I I I}, \mathcal{Z}_{I I I}^{D_{7}}, \mathcal{Z}_{I I} \\
0 & \text { for } \mathcal{Z}_{I I I}^{D_{8}}, \mathcal{Z}_{I}
\end{array}\right. \\
& D_{1}=\left\{\begin{array}{cc}
\text { arbitrary, }, & \text { for } \mathcal{Z}_{V}, \mathcal{Z}_{I V}, \\
0, & \text { for } \mathcal{Z}_{I I I}, \mathcal{Z}_{I I I}^{D_{7}}, \mathcal{Z}_{I I I}^{D_{8}}, \mathcal{Z}_{I I}, \mathcal{Z}_{I} .
\end{array}\right.
\end{aligned}
$$

In Theorem 6.2, we prove that the spherical sub-algebras of our confluent Cherednik algebras are isomorphic to the corresponding confluent Zhedanov algebras quotiented over their Casimir.

In order to prove Theorem 6.2 we provide an embedding for each (confluent) Cherednik algebras $\mathcal{H}, \mathcal{H}_{V}, \mathcal{H}_{I V}, \mathcal{H}_{I I I}, \mathcal{H}_{I I}$ and $\mathcal{H}_{I}$ in $\operatorname{Mat}\left(2, \mathbb{T}_{q}\right)$, where $\mathbb{T}_{q}$ is the rank 2 quantum torus, i.e. the ring of Laurent polynomials in $x, y, q$ with commutation relations $x y=q y x, q x=x q, q y=y q$ (see Theorems 5.2,5.3,5.4,5.5,5.6). 
These embeddings show that all of these algebras are Azumaya of rank 2 (for $\mathcal{H}$, already appeared in [35] $)^{2}$.

Finally, we give a faithful representation of the confluent Zhedanov algebras $\mathcal{Z}_{V}, \mathcal{Z}_{I I I}, \mathcal{Z}_{I I I}^{D_{7}}, \mathcal{Z}_{I I I}^{D_{8}}$ on the space of symmetric Laurent polynomials and of the confluent Zhedanov algebras $\mathcal{H}_{I V}, \mathcal{H}_{I I}$ and $\mathcal{H}_{I}$ on the space of polynomials and prove that specific elements of the q-Askey scheme arise as eigenvectors of one of the operators in such representations. ${ }^{3}$ There results are schematically resumed in figure 1 .
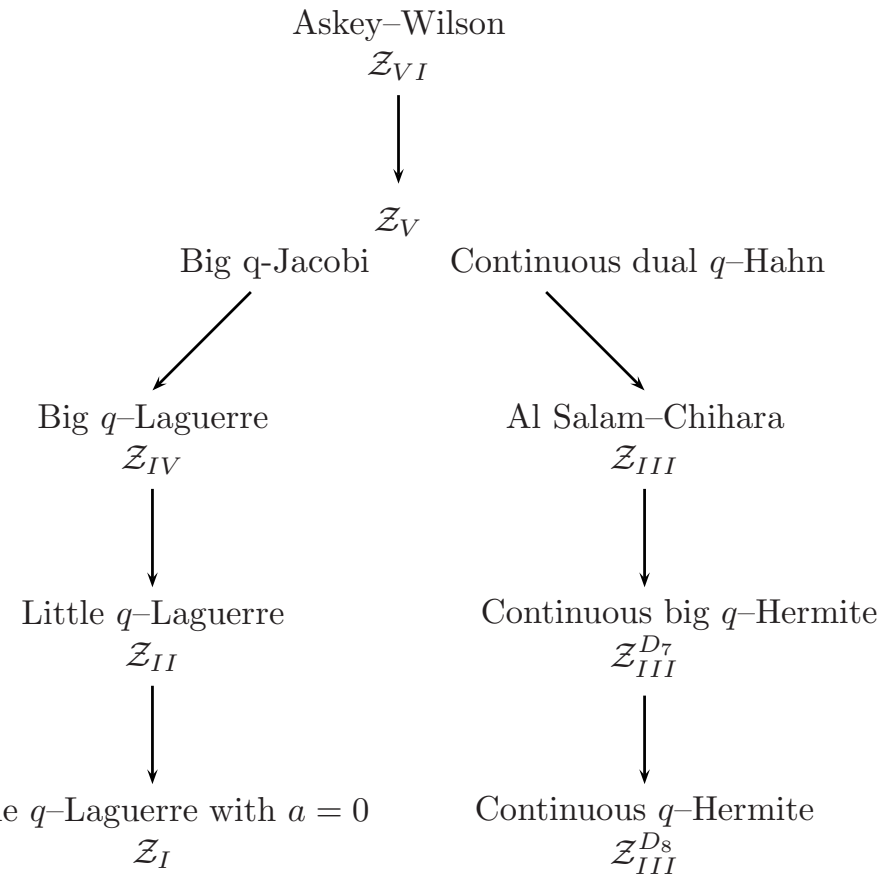

FiguRE 1. The confluence scheme for the Zhedanov algebras and the polynomials in the q-Askey scheme. Note that each confluent Zhedanov algebra is labelled by the Painlevé equation whose the monodromy manifold arises as its semiclassical limit.

Note that for $\mathcal{Z}_{V}$ we have two different faithful representations corresponding to the continuous dual $q$-Hahn polynomials and to the big $q$-Jacobi polynomials, which is due to an algebra automorphism of $\mathcal{H}$ which under confluence produces an algebra isomorphic to $\mathcal{H}_{V}$ as described in sub-section 3.1 and in Lemma 6.7. This algebra isomorphism reflects the duality between continuous dual $q-$ Hahn polynomials and big $q$-Jacobi polynomials [27]. The author conjectures that all families of polynomials in the left side of the q-Askey scheme can be obtained with a similar construction from confluences of the algebra automorphisms of $\mathcal{H}$.

\footnotetext{
${ }^{2}$ The author is grateful to Pavel Etingof for this observation.

${ }^{3}$ The basic representation for the algebras $\mathcal{Z}_{V}, \mathcal{Z}_{I I I}, \mathcal{Z}_{I I I}^{D_{7}}, \mathcal{Z}_{I I I}^{D_{8}}$ can be found in [29].
} 
Remark 1.5. In [8], Cherednik introduced a Whittaker limit on difference spherical functions and correspondingly introduced a notion of nil-DAHA for the root system $A_{n}$. The confluent Cherednik algebras defined in the current paper are a concatenation of Whittaker-type limits in the case of root system $C_{1}{ }^{4}$

This paper is organised as follows: In Section 2, we recall some background material on the theory of the Cherednik algebra of type $\check{C}_{1} C_{1}$. In Section 3 , we explain how to derive our confluent Cherednik algebras and give some equivalent presentations for them. In Section 4, we discuss the spherical sub-algebras and show that the generators satisfy a cubic relation which in the limit coincides with the monodromy manifolds of the corresponding Painlevé equations. In Section 5 we provide an embedding for each (confluent) Cherednik algebras $\mathcal{H}, \mathcal{H}_{V}, \mathcal{H}_{I V}, \mathcal{H}_{I I I}, \mathcal{H}_{I I}$ and $\mathcal{H}_{I}$ in $\operatorname{Mat}\left(2, \mathbb{T}_{q}\right)$, where $\mathbb{T}_{q}$ is the rank 2 quantum torus. In Section 6 , we prove that each spherical sub-algebra is isomorphic to the corresponding confluent Zhedanov algebra quotiented by its Casimir and show that the latter act as symmetries of some elements of the q-Askey scheme. In the appendix we list the Painlevé monodromy manifolds.

\section{Notation And BACKground on the CheredniK ALgebra of type $\check{C}_{1} C_{1}$}

In this section we recall some background material on the theory of the Cherednik algebra of type $\check{C}_{1} C_{1}$ and the relation due to Koornwinder [24, 25] between its spherical sub-algebra and Askey-Wilson polynomials. Throughout this section we assume $q^{m} \neq 1$, for all $m \in \mathbb{Z}$.

2.1. Automorphisms of the Cherednik algebra of type $\check{C}_{1} C_{1}$. The automorphisms of the Cherednik algebra of type $\check{C}_{1} C_{1}$ were studied in [33, 43]. Here we list the ones that will be used in this paper:

Proposition 2.1. The following transformations are automorphisms of the Cherednik algebra of type $\check{C}_{1} C_{1}$ :

$$
\begin{aligned}
& \beta:\left\{\begin{aligned}
T_{0} & \rightarrow-\frac{q}{c} W T_{0}-\left(1+\frac{d}{c}\right), \\
T_{1} & \rightarrow T_{1}, \\
X & \rightarrow X, \\
a & \rightarrow a, \\
b & \rightarrow b, \\
c & \rightarrow \frac{q}{c}, \\
d & \rightarrow d,
\end{aligned}\right. \\
& \gamma:\left\{\begin{aligned}
T_{0} & \rightarrow b T_{1}^{-1} W, \\
T_{1} & \rightarrow T_{1}, \\
X & \rightarrow \sqrt{\frac{a b c d}{q}} T_{1}^{-1} W T_{0}^{-1} X, \\
a & \rightarrow \sqrt{\frac{a b c d}{q}}, \\
b & \rightarrow-\sqrt{\frac{q a b}{c d}} \\
c & \rightarrow-\sqrt{\frac{q b c}{a d}}, \\
d & \rightarrow \sqrt{\frac{q b d}{a c}} .
\end{aligned}\right.
\end{aligned}
$$

\footnotetext{
${ }^{4}$ The author is grateful to P. Etingof for pointing this out to her.
} 
It is worth noticing that in the limit $q \rightarrow 1$ these automorphisms correspond to the action of the braid group on the monodromy matrices associated to the sixth Painlevé equation [30].

2.2. Zhedanov algebra and Askey Wilson polynomials. Let us remind the definition of Zhedanov algebra:

Definition 2.2. [52] Let $B, C_{0}, C_{1}, D_{0}, D_{1} \in \mathbb{C}$. The Zhedanov algebra $\mathcal{Z}$ is the algebra generated by three elements $K_{0}, K_{1}$ and $K_{2}$ which satisfy the following relations:

$$
\begin{array}{r}
q^{\frac{1}{2}} K_{0} K_{1}-q^{-\frac{1}{2}} K_{1} K_{0}=K_{2}, \\
q^{\frac{1}{2}} K_{1} K_{2}-q^{-\frac{1}{2}} K_{2} K_{1}=B K_{1}+C_{0} K_{0}+D_{0}, \\
q^{\frac{1}{2}} K_{2} K_{0}-q^{-\frac{1}{2}} K_{0} K_{2}=B K_{0}+C_{1} K_{1}+D_{1} .
\end{array}
$$

Note that this algebra admits the following Casimir

$$
\begin{aligned}
& \mathcal{C}:=q^{-\frac{1}{2}}\left(1-q^{2}\right) K_{0} K_{1} K_{2}+q K_{2}^{2}+B\left(K_{0} K_{1}+K_{1} K_{0}\right)+q C_{0} K_{0}^{2}+ \\
& +\frac{C_{1}}{q} K_{1}^{2}+(1+q) D_{0} K_{0}+\left(1+\frac{1}{q}\right) D_{1} K_{1} .
\end{aligned}
$$

The Zhedanov algebra depends on 5 parameters, but we can choose two of them arbitrarily, for example $C_{1}$ and $C_{0}$ by rescaling the generators. The quotient algebra $\mathcal{Z}\left(\mathcal{C}_{0}\right)=\mathcal{Z} /\left\langle\mathcal{C}=\mathcal{C}_{0}\right\rangle$ therefore depends on 4 independent parameters (one of them being the value of $\mathcal{C}_{0}$ ).

In [24] Koornwinder defined an embedding of the central extension of the Zhedanov algebra $\mathcal{Z}\left(\mathcal{C}_{0}\right)$, into the Cherednik algebra $\mathcal{H}$ of type $\check{C}_{1} C_{1}$. This result was then generalised to the universal Askey-Wilson algebra defined in [44] and [45].

Without going into too much detail, let us recall the main ingredients of Koornwinder embedding. Let us express the Zhedanov algebra structure constants by the parameters $a, b, c, d$ :

$$
\begin{aligned}
& B=\frac{(q-1)^{2}}{q}\left(\left(1+\frac{a b}{q}\right)\left(\frac{d}{c}+1\right) c+\left(\frac{b}{a}+1\right)\left(1+\frac{c d}{q}\right) a\right), \\
& C_{0}=\left(q-\frac{1}{q}\right)^{2}, \quad C_{1}=\frac{a b c d}{q}\left(q-\frac{1}{q}\right)^{2} \\
& D_{0}=-\frac{(q+1)(q-1)^{2}}{q}\left(\left(\frac{b}{a}+1\right)\left(\frac{d}{c}+1\right) \frac{a c}{q}+\left(1+\frac{a b}{q}\right)\left(1+\frac{c d}{q}\right)\right), \\
& D_{1}=-\frac{(q+1)(q-1)^{2}}{q^{2}}\left(\left(\frac{b}{a}+1\right)\left(1+\frac{a b}{q}\right) a c d+\left(\frac{d}{c}+1\right)\left(1+\frac{c d}{q}\right) a b c\right),
\end{aligned}
$$

then $\mathcal{Z}\left(\mathcal{C}_{0}\right)$ admits the following representation on the space $\mathcal{L}_{\text {sym }}$ of symmetric Laurent polynomials $[24,26]$ :

$$
\begin{aligned}
\left(K_{1} f\right)[x]:= & \left(x+\frac{1}{x}\right) f[x], \\
\left(K_{0} f\right)[x]:= & \frac{(1-a x)(1-b x)(1-c x)(1-d x)}{\left(1-x^{2}\right)\left(1-q x^{2}\right)}(f[q x]-f[x])+ \\
& +\frac{(a-x)(b-x)(c-x)(d-x)}{\left(1-x^{2}\right)\left(q-x^{2}\right)}\left(f\left[q^{-1} x\right]-f[x]\right)+
\end{aligned}
$$




$$
+\left(1+\frac{a b c d}{q}\right) f[x] .
$$

We don't list here the representation for the operator $K_{2}$ as this can be obtained by applying relation (2.54).

The Askey Wilson polynomials (we write them here in monic form like in [24]):

$$
P_{n}(x ; a, b, c, d):=\frac{(a b, a c, a d ; q)_{n}}{a^{n}\left(a b c d q^{n-1} ; q\right)_{n}}{ }_{4} \phi_{3}\left(\begin{array}{c}
q^{-n}, q^{n-1} a b c d, a x, a x^{-1} \\
a b, a c, a d
\end{array} ; q, q\right),
$$

are eigenfunctions of the $K_{0}$ operator:

$$
K_{0} P_{n}=\left(q^{-n}+a b c d q^{n-1}\right) P_{n} .
$$

The reduction from the space $\mathcal{L}$ of Laurent polynomials to the space $\mathcal{L}_{\text {sym }}$ of symmetric Laurent polynomials is due to the action of the symmetriser of $\mathcal{H}$ :

$$
e:=\frac{1+i \sqrt{a b} \check{V}_{1}}{1-a b}
$$

which allowed Koornwinder to establish the isomorphism between a central extension $\widetilde{\mathcal{Z}}\left(\mathcal{C}_{0}\right)$ and the spherical sub-algebra $e \mathcal{H} e$ of $\mathcal{H}$. We discuss this result and the link with the PVI monodromy manifold in the next subsection.

2.3. An important cubic relation and the spherical sub-algebra $e \mathcal{H} e$. The following result plays an important role in this paper:

Proposition 2.3. The following three elements:

$$
\begin{gathered}
X_{1}=X+W, \quad X_{2}=\sqrt{\frac{a b c d}{q}} T_{0}^{-1} T_{1}^{-1}+\sqrt{\frac{q}{a b c d}} T_{1} T_{0}, \\
X_{3}=q \sqrt{a b c d}\left(\frac{1}{a b c d} W T_{0} T_{1}+\frac{1}{a b c d} X T_{1} T_{0}+\left(\frac{1}{a}+\frac{1}{b}\right) \frac{1}{c d} T_{0}+\left(\frac{1}{c}+\frac{1}{d}\right) \frac{1}{a b q} T_{1}\right),
\end{gathered}
$$

commute with $e$ and satisfy the quantum commutation relations:

$$
\begin{gathered}
q^{\frac{1}{2}} X_{2} X_{1}-q^{-\frac{1}{2}} X_{1} X_{2}=\left(q-\frac{1}{q}\right) X_{3}- \\
-\left(q^{\frac{1}{2}}-q^{-\frac{1}{2}}\right)\left(\bar{k}_{0} \bar{k}_{1}-\bar{u}_{0}\left(\frac{i}{\sqrt{q a b}} T_{1}+i \sqrt{q a b} T_{1}^{-1}\right)\right), \\
q^{\frac{1}{2}} X_{3} X_{2}-q^{-\frac{1}{2}} X_{2} X_{3}=\left(q-\frac{1}{q}\right) X_{1}- \\
-\left(q^{\frac{1}{2}}-q^{-\frac{1}{2}}\right)\left(\bar{k}_{0} \bar{u}_{0}-\bar{k}_{1}\left(\frac{i}{\sqrt{q a b}} T_{1}+i \sqrt{q a b} T_{1}^{-1}\right)\right), \\
q^{\frac{1}{2}} X_{1} X_{3}-q^{-\frac{1}{2}} X_{3} X_{1}=\left(q-\frac{1}{q}\right) X_{2}- \\
-\left(q^{\frac{1}{2}}-q^{-\frac{1}{2}}\right)\left(\bar{k}_{1} \bar{u}_{0}-\bar{k}_{0}\left(\frac{i}{\sqrt{q a b}} T_{1}+i \sqrt{q a b} T_{1}^{-1}\right)\right),
\end{gathered}
$$

and the quantum cubic relation:

$$
\begin{aligned}
q^{\frac{1}{2}} X_{2} X_{1} X_{3}-q X_{2}^{2}-\frac{1}{q} X_{1}^{2}-q X_{3}^{2} & +\sqrt{q}\left(\bar{k}_{1} \bar{u}_{0}-\bar{k}_{0}\left(\frac{i}{\sqrt{q a b}} T_{1}+i \sqrt{q a b} T_{1}^{-1}\right)\right) X_{2}+ \\
& +\frac{1}{\sqrt{q}}\left(\bar{u}_{0} \bar{k}_{0}-\bar{k}_{1}\left(\frac{i}{\sqrt{q a b}} T_{1}+i \sqrt{q a b} T_{1}^{-1}\right)\right) X_{1}+
\end{aligned}
$$




$$
\begin{array}{r}
+\sqrt{q}\left(\bar{k}_{0} \bar{k}_{1}-\bar{u}_{0}\left(\frac{i}{\sqrt{q a b}} T_{1}+i \sqrt{q a b} T_{1}^{-1}\right)\right) X_{3}+ \\
+\bar{k}_{0}^{2}+\bar{k}_{1}^{2}+\bar{u}_{0}^{2}-\bar{u}_{1}^{2}+2\left(q+\frac{1}{q}\right)+\left(\frac{q+1}{\sqrt{q}} \bar{u}_{1}-\bar{k}_{0} \bar{k}_{1} \bar{u}_{0}\right)\left(\frac{i}{\sqrt{q a b}} T_{1}+i \sqrt{q a b} T_{1}^{-1}\right),
\end{array}
$$

where:

$$
\begin{aligned}
& \bar{u}_{0}=u_{0}-\frac{1}{u_{0}}=i \sqrt{\frac{d}{c}}+i \sqrt{\frac{c}{d}}, \quad \bar{k}_{0}=k_{0}-\frac{1}{k_{0}}=i \sqrt{\frac{c d}{q}}+i \sqrt{\frac{q}{c d}}, \\
& \bar{u}_{1}=u_{1}-\frac{1}{u_{1}}=i \sqrt{a b}+i \frac{1}{\sqrt{a b}}, \quad \bar{k}_{1}=k_{1}-\frac{1}{k_{1}}=i \sqrt{\frac{b}{a}}+i \sqrt{\frac{a}{b}} .
\end{aligned}
$$

Proof. Relations (2.62) are proved in [19] (see also [10]). The only proof the author could find of this cubic relation (2.65) is in [10] for the case $\bar{u}_{0}=\bar{u}_{1}=\bar{k}_{0}=\bar{k}_{1}=0$. To prove it in the generic case, we use the following substitutions which can be easily be derived from $(1.2,1.3,1.4)$ and $(1.5)$ :

$$
\begin{array}{r}
T_{1}^{2}=-(a b+1) T_{1}-a b, \quad T_{0}^{2}=-(c d / q+1) T_{0}-\frac{c d}{q} \\
T_{1} X=W T_{1}+(1+a b) W-(a+b), \\
T_{1} W=X T_{1}-(1+a b) W+a+b, \\
T_{0} X=q W T_{0}+c+d-(1+c d / q) X, \\
T_{0} W=\frac{1}{q} X T_{0}+\frac{1}{q}(1+c d / q) X-\frac{1}{q}(c+d) .
\end{array}
$$

Using these relations recursively, it is a straightforward computation to eliminate all higher powers in $T_{0}$ and $T_{1}$ and to bring all $X$ and $W$ to the left and eventually obtain the statement.

The following lemma characterises the spherical-subalgebra $e \mathcal{H e}$ :

Corollary 2.4. $[25,10,19]$ The elements $\hat{X}_{i}=e X_{i} e, i=1,2,3$, where $X_{1}, X_{2}, X_{3}$ are defined by (2.61), generate the spherical sub-algebra $e \mathcal{H} e$, they satisfy the quantum commutation relations:

$$
\begin{aligned}
& q^{\frac{1}{2}} \hat{X}_{2} \hat{X}_{1}-q^{-\frac{1}{2}} \hat{X}_{1} \hat{X}_{2}=\left(q-\frac{1}{q}\right) \hat{X}_{3}-\left(q^{\frac{1}{2}}-q^{-\frac{1}{2}}\right) \omega_{3} e \\
& q^{\frac{1}{2}} \hat{X}_{3} \hat{X}_{2}-q^{-\frac{1}{2}} \hat{X}_{2} \hat{X}_{3}=\left(q-\frac{1}{q}\right) \hat{X}_{1}-\left(q^{\frac{1}{2}}-q^{-\frac{1}{2}}\right) \omega_{1} e \\
& q^{\frac{1}{2}} \hat{X}_{1} \hat{X}_{3}-q^{-\frac{1}{2}} \hat{X}_{3} \hat{X}_{1}=\left(q-\frac{1}{q}\right) \hat{X}_{2}-\left(q^{\frac{1}{2}}-q^{-\frac{1}{2}}\right) \omega_{2} e
\end{aligned}
$$

and the following cubic relation:

$$
q^{\frac{1}{2}} \hat{X}_{2} \hat{X}_{1} \hat{X}_{3}-q \hat{X}_{2}^{2}-q^{-1} \hat{X}_{1}^{2}-q \hat{X}_{3}^{2}+\sqrt{q} \omega_{2} \hat{X}_{2}+\frac{1}{\sqrt{q}} \omega_{1} \hat{X}_{1}+\sqrt{q} \omega_{3} \hat{X}_{3}-\omega_{4} e=0 .
$$

where

$$
\begin{aligned}
& \omega_{1}=\bar{u}_{0} \bar{k}_{0}+i \bar{k}_{1}\left(\sqrt{\frac{a b}{q}}+\sqrt{\frac{q}{a b}}\right), \\
& \omega_{2}=\bar{k}_{1} \bar{u}_{0}+i \bar{k}_{0}\left(\sqrt{\frac{a b}{q}}+\sqrt{\frac{q}{a b}}\right),
\end{aligned}
$$




$$
\begin{array}{r}
\omega_{3}=\bar{k}_{0} \bar{k}_{1}+i \bar{u}_{0}\left(\sqrt{\frac{a b}{q}}+\sqrt{\frac{q}{a b}}\right), \\
\omega_{4}=\bar{k}_{0}^{2}+\bar{k}_{1}^{2}+\bar{u}_{0}^{2}-\left(\sqrt{\frac{a b}{q}}+\sqrt{\frac{q}{a b}}\right)^{2}-i \bar{k}_{0} \bar{k}_{1} \bar{u}_{0}\left(\sqrt{\frac{a b}{q}}+\sqrt{\frac{q}{a b}}\right)+\frac{(1+q)^{2}}{q},
\end{array}
$$

where $\bar{k}_{0}, \bar{k}_{1}, \bar{u}_{0}, \bar{u}_{1}$ are defined in $(2.66)$.

Lemma 2.5. [35] In the limit $q \rightarrow 1, X_{1}, X_{2}, X_{3}$ satisfy the following cubic relation:

$$
\begin{aligned}
& X_{1} X_{2} X_{3}-X_{1}^{2}-X_{2}^{2}-X_{3}^{2}+\left(\bar{u}_{0} \bar{k}_{0}+\bar{u}_{1} \bar{k}_{1}\right) X_{1}+\left(\bar{k}_{1} \bar{u}_{0}+\bar{k}_{0} \bar{u}_{1}\right) X_{2}+ \\
& \quad+\left(\bar{k}_{0} \bar{k}_{1}+\bar{u}_{0} \bar{u}_{1}\right) X_{3}+\bar{k}_{0}^{2}+\bar{k}_{1}^{2}+\bar{u}_{0}^{2}+\bar{u}_{1}^{2}-\bar{k}_{0} \bar{k}_{1} \bar{u}_{0} \bar{u}_{1}+4=0 .
\end{aligned}
$$

Remark 2.6. This cubic is also known as the monodromy manifold of the sixth Painlevé equation (see Appendix A). In this paper we will obtain similar cubic relations for the spherical subalgebras of each confluent Cherednik algebra and we will show that in the classical limit each of these cubic relations produces the monodromy manifold of the corresponding Painlevé equation.

\section{Derivation and first properties of the confluent Cherednik ALGEBRAS}

The procedure to derive the confluent Cherednick algebras given in Definition 1.1 can be resumed in two main roads, i.e. $\mathcal{H}_{V I} \rightarrow \mathcal{H}_{V} \rightarrow \mathcal{H}_{I I I} \rightarrow \mathcal{H}_{I I I}^{D_{7}} \rightarrow \mathcal{H}_{I I I}^{D_{8}}$ and $\mathcal{H}_{V I} \rightarrow \mathcal{H}_{V} \rightarrow \mathcal{H}_{I V} \rightarrow \mathcal{H}_{I I} \rightarrow \mathcal{H}_{I}$. In the first road we make $T_{0}$ more and more singular, then we make $T_{1}$ more and more singular, while keeping $X$ and $W$ invertible. In the second road we make $X$ and $W$ singular, then $T_{0}$ more and more singular. To reach $\mathcal{H}_{I}$ however, we need to swap to the Noumi-Stokman presentation of the algebra $\mathcal{H}_{V I}$ otherwise we end up by loosing a generator. For this reason in this section we start by giving the Noumi-Stokman presentation for the algebras $\mathcal{H}_{V I}, \mathcal{H}_{V}, \mathcal{H}_{I V}, \mathcal{H}_{I I I} \mathcal{H}_{I I}$, then we explain our derivation procedure more in detail.

Remark 3.1. As pointed out to the author by T. Koornwinder, there always is a degree of arbitrariness in such a confluence procedure. However there are two very strong mechanisms to remove such arbitrariness: the first one is that many confluences lead to algebras with too many relations and therefore are to be discarded. The second is that many different confluences give rise to algebras which are equivalent by an algebra isomorphism as in the case of $\mathcal{H}_{V}$. In this paper we impose a specific degeneration scheme for the cubic relations satisfied by the generators of the spherical sub-algebras such that in the limit they give rise to the Poisson relations on the monodromy manifolds of the Painlevé equations (see Section 4).

Theorem 3.2. Let $k_{1}, u_{0}, u_{1}, q \in \mathbb{C}^{\star}$. The confluent Cherednik algebras $\mathcal{H}_{V}, \mathcal{H}_{I V}$, $\mathcal{H}_{I I I}, \mathcal{H}_{I I}$ are isomorphic as algebras to the associative $\mathbb{C}$-algebra with generators $V_{0}, \breve{V}_{0}, V_{1}, \check{V}_{1}$ and relations:

- $\mathcal{H}_{V}$ :

$$
\begin{aligned}
V_{0}^{2}+V_{0} & =0, \\
\left(V_{1}-k_{1}\right)\left(V_{1}+k_{1}^{-1}\right) & =0,
\end{aligned}
$$




$$
\begin{array}{r}
\check{V}_{0}^{2}+u_{0}^{-1} \check{V}_{0}=0, \\
\left(\check{V}_{1}-u_{1}\right)\left(\check{V}_{1}+u_{1}^{-1}\right)=0, \\
q^{1 / 2} \check{V}_{1} V_{1} V_{0}=\check{V}_{0}+u_{0}^{-1}, \\
q^{1 / 2} \check{V}_{0} \check{V}_{1} V_{1}=V_{0}+1 .
\end{array}
$$

- $\mathcal{H}_{I V}$ :

- $\mathcal{H}_{I I I}$ :

- $\mathcal{H}_{I I}$ :

$$
\begin{aligned}
& V_{0}^{2}+V_{0}=0, \\
& V_{1}^{2}+V_{1}=0, \\
& \check{V}_{0}^{2}+\frac{1}{u_{0}} \check{V}_{0}=0, \\
&\left(\check{V}_{1}-u_{1}\right)\left(\check{V}_{1}+u_{1}^{-1}\right)=0, \\
& q^{1 / 2} \check{V}_{1} V_{1} V_{0}=\check{V}_{0}+u_{0}^{-1}, \\
& \check{V}_{0} \check{V}_{1} V_{1}=0, \\
& V_{0} \check{V}_{0}=0 .
\end{aligned}
$$

Proof. To avoid repeating the proof three times we use the following conventions:

$$
\begin{aligned}
& u_{0}=\sqrt{q} \text { for } \mathcal{H}_{I I I} \\
& u_{0}=1 \text { for } \mathcal{H}_{I I} \\
& k_{1}=1 \text { for } \mathcal{H}_{I V}, \mathcal{H}_{I I} .
\end{aligned}
$$

It is enough to give relations between the generators $V_{0}, V_{1}, \check{V}_{0}, \check{V}_{1}$ and $X, W, T_{0}, T_{1}$ :

$$
\begin{aligned}
T_{0} & =V_{0}, \quad T_{1}=u_{1} \check{V}_{1}, \quad W=\check{V}_{1} V_{1}, \\
X & =\left\{\begin{array}{lc}
\left(V_{1}+k_{1}^{-1}-k_{1}\right)\left(\check{V}_{1}+u_{1}^{-1}-u_{1}\right), & \text { for } \mathcal{H}_{V} \text { and } \mathcal{H}_{I I I} \\
\left(V_{1}+1\right)\left(\check{V}_{1}+u_{1}^{-1}-u_{1}\right), & \text { for } \mathcal{H}_{I V},
\end{array}\right.
\end{aligned}
$$


and for the parameters:

$$
a=-\frac{u_{1}}{k_{1}}, \quad b=k_{1} u_{1}, \quad c=-q^{\frac{1}{2}} \frac{1}{u_{0}} .
$$

Viceversa:

$$
V_{0}=T_{0}, \quad \check{V_{1}}=\frac{1}{u_{1}} T_{1}, \quad \check{V_{0}}=q^{1 / 2} W T_{0}-\frac{1}{u_{0}}, \quad V_{1}=u_{1} T_{1}^{-1} W,
$$

where

$$
T_{1}^{-1}=-\frac{1}{a b} T_{1}-\left(1+\frac{1}{a b}\right) .
$$

3.1. Derivation of $\mathcal{H}_{V}$. The derivation procedure is more transparent in NoumiStokman representation. Start from $\mathcal{H}$ and choose to rescale $V_{0}$ and $\check{V}_{0}$. Then (1.40) will become singular, so before taking the limit we replace it by:

$$
\sqrt{q} \check{V}_{1} V_{1} V_{0}=\check{V}_{0}-\bar{u}_{0}, \quad \sqrt{q} \check{V}_{0} \check{V}_{1} V_{1}=V_{0}-\bar{k}_{0} .
$$

Now rescale: $V_{0} \rightarrow \frac{1}{\varepsilon} V_{0}, \check{V}_{0} \rightarrow \frac{1}{\varepsilon} \check{V}_{0}, k_{0} \rightarrow \varepsilon$, and $u_{0} \rightarrow \varepsilon u_{0}$. Then the defining relations $(1.36,1.38,1.40)$ become

$$
\begin{array}{ll}
\frac{1}{\varepsilon^{2}}\left(V_{0}-\varepsilon^{2}\right)\left(V_{0}+k_{0}^{-1}\right)=0, & \frac{1}{\varepsilon^{2}}\left(\check{V}_{0}-\varepsilon^{2} u_{0}\right)\left(\check{V}_{0}+u_{0}^{-1}\right)=0, \\
\frac{1}{\varepsilon} \sqrt{q} \check{V}_{1} V_{1} V_{0}=\frac{1}{\varepsilon} \check{V}_{0}+\frac{1}{\varepsilon} u_{0}^{-1}, & \frac{1}{\varepsilon} \sqrt{q} \check{V}_{0} \check{V}_{1} V_{1}=\frac{1}{\varepsilon} V_{0}+\frac{1}{\varepsilon} k_{0}^{-1} .
\end{array}
$$

and in the limit $\varepsilon \rightarrow 0$ we obtain $\mathcal{H}_{V}$. Observe that the new $V_{0}$ and $\check{V}_{0}$ are no longer invertible and $q$ has not been rescaled - differently from the case of rational Cherednik algerbas $[2,18]$.

We can derive $\mathcal{H}_{V}$ also in another way: choose to rescale $V_{1}$ and $\check{V}_{0}$. Then (1.40) will become singular and needs to be replaced by:

$$
\sqrt{q} V_{0} \check{V}_{0} \check{V}_{1}=\check{V}_{1}-\bar{k}_{1} \quad \sqrt{q} \check{V}_{1} V_{1} V_{0}=\check{V}_{0}-\bar{u}_{0}, .
$$

Now rescale: $V_{1} \rightarrow \frac{1}{\varepsilon} V_{1}, \check{V}_{0} \rightarrow \frac{1}{\varepsilon} \check{V}_{0}, k_{1} \rightarrow-\frac{1}{\varepsilon}$, and $u_{0} \rightarrow \varepsilon u_{0}$. Then the defining relations $(1.37,1.38,1.40)$ become

$$
\begin{array}{r}
\frac{1}{\varepsilon^{2}}\left(V_{1}+1\right)\left(V_{1}-\varepsilon^{2}\right)=0, \\
\frac{1}{\varepsilon^{2}}\left(\check{V}_{0}-\varepsilon^{2} u_{0}\right)\left(\check{V}_{0}+u_{0}^{-1}\right)=0, \\
\frac{1}{\varepsilon} \sqrt{q} V_{0} \check{V}_{0} \check{V}_{1}=\frac{1}{\varepsilon} V_{1}+\frac{1}{\varepsilon} \quad \frac{1}{\varepsilon} \sqrt{q} \check{V}_{1} V_{1} V_{0}=\frac{1}{\varepsilon} \check{V_{0}}+\frac{1}{\varepsilon} u_{0}^{-1} .
\end{array}
$$

By taking the limit $\varepsilon \rightarrow 0$ we obtain the following algebra $\mathcal{H}_{V}^{\gamma}$ :

$$
\begin{aligned}
&\left(V_{0}-k_{0}\right)\left(V_{0}+k_{0}^{-1}\right)=0 \\
&\left(V_{1}+1\right) V_{1}=0 \\
& \check{V}_{0}^{2}+u_{0}^{-1} \check{V}_{0}=0 \\
&\left(\check{V}_{1}-u_{1}\right)\left(\check{V}_{1}+u_{1}^{-1}\right)=0 \\
& q^{1 / 2} \check{V}_{1} V_{1} V_{0}=\check{V}_{0}+u_{0}^{-1} \\
& q^{1 / 2} V_{0} \check{V}_{0} \check{V}_{1}=V_{1}+1
\end{aligned}
$$


This algebra is the image of $\mathcal{H}_{V}$ in the (3.73-3.78) presentation by the following isomorphism $\gamma$ :

$$
\gamma\left(\check{V}_{1}, V_{1}, V_{0}, \check{V}_{0}\right)=\left(\check{V}_{1}, V_{1} V_{0} V_{1}^{-1}, V_{1}, \check{V}_{0}\right), \quad \gamma\left(u_{1}, k_{1}, k_{0}, u_{0}\right)=\left(u_{1}, k_{0}^{-1}, k_{1}, u_{0}\right),
$$

where we pick $k_{0}=1$. In Sahi presentation the algebra $\mathcal{H}_{V}^{\gamma}$ takes the following form:

$$
\begin{array}{r}
X W=W X=0, \\
\left(T_{1}-a^{2}\right)\left(T_{1}+1\right)=0, \\
\left(T_{0}+\frac{b c}{q}\right)\left(T_{0}+1\right)=0, \\
T_{1} X-a=W\left(T_{1}+1-a^{2}\right), \\
q T_{0} W+c=X\left(T_{0}+1+\frac{b c}{q}\right),
\end{array}
$$

which is the image of $\mathcal{H}_{V}$ under the isomorphism:

$$
\begin{aligned}
& \gamma\left(T_{0}, T_{1}, X, W\right)=\left(b T_{1}^{-1} W, T_{1}, i \sqrt{a b} T_{1}^{-1} W\left(T_{0}+1\right) X,-\frac{i}{\sqrt{a b}} W T_{0} X T_{1}\right), \\
& \gamma(a, b, c)=\left(i \sqrt{a b}, i c \sqrt{\frac{b}{a}},-i \frac{q}{c} \sqrt{\frac{b}{a}}\right) .
\end{aligned}
$$

Note that this fact has an interesting consequence in terms of $q$-polynomials: we shall see in Section 6 that the spherical sub-algebra of $\mathcal{H}_{V}$ acts as symmetries both on the continuous dual $q$-Hahn polynomials and the big $q$-Jacobi polynomials.

3.2. Derivation of $\mathcal{H}_{I V}, \mathcal{H}_{I I I}, \mathcal{H}_{I I}, \mathcal{H}_{I}$. Again, the derivation procedure is more transparent in Noumi-Stokman representation. In each case we do a rescaling and then take the limit $\varepsilon \rightarrow 0$ :

The algebra $\mathcal{H}_{I V}$ is obtained from $\mathcal{H}_{V}^{\gamma}$ by rescaling $V_{0} \rightarrow \frac{1}{\varepsilon} V_{0}$ and $\check{V}_{0} \rightarrow \frac{1}{\varepsilon} \check{V}_{0}$, $k_{1} \rightarrow \varepsilon$, and $u_{0} \rightarrow \varepsilon u_{0}$.

The algebra $\mathcal{H}_{I I I}$ is obtained from $\mathcal{H}_{V}$ by rescaling $V_{0} \rightarrow \frac{1}{\varepsilon} V_{0}, \check{V}_{0} \rightarrow \frac{1}{\varepsilon} \check{V}_{0}$ and $u_{0} \rightarrow \varepsilon \sqrt{q}$.

The algebra $\mathcal{H}_{I I}$ is obtained from $\mathcal{H}_{I V}$ by rescaling $\check{V}_{0} \rightarrow \frac{1}{\varepsilon} \check{V}_{0}, V_{1} \rightarrow \frac{1}{\varepsilon} V_{1} u_{0} \rightarrow \varepsilon$.

The algebra $\mathcal{H}_{I}$ is obtained from $\mathcal{H}_{I I}$ by rescaling $\check{V}_{1} \rightarrow \frac{1}{\varepsilon} \check{V}_{1}, \check{V}_{0} \rightarrow \frac{1}{\varepsilon} \check{V}_{0}$ and $u_{1} \rightarrow \varepsilon$.

3.3. Derivation of $\mathcal{H}_{I I I^{D_{7}}}$, and $\mathcal{H}_{I I^{I^{D_{8}}}}$. In this case we don't have a NoumiStokman representation. We start form $\mathcal{H}_{I I I}$ in the presentation $(1.16 \ldots, 1.20)$. Rewrite relation (1.19) by using (1.17):

$$
T_{1} X+(a+b)=W\left(T_{1}-(a+b)\right) .
$$

Then take the limit as $b \rightarrow 0$ to obtain $(1.21, \ldots 1.25)$.

Analogously, starting from $(1.21, \ldots 1.25)$ and taking $a \rightarrow 0$, we obtain the $\mathcal{H}_{I I I^{D_{8}}}$ algebra.

\section{Confluent spherical sub-Algebras and Painlevé Cubics}

In this section we give the confluent version of the results of section 2.3 for each algebra $\mathcal{H}_{V}, \mathcal{H}_{I V}, \mathcal{H}_{I I I}, \mathcal{H}_{I I I^{D_{7}}}, \mathcal{H}_{I I I^{D_{8}}}, \mathcal{H}_{I I}, \mathcal{H}_{I}$ to produce in each case the appropriate cubic surface as limit of the spherical subalgebras. In each case we will 
select a idempotent element $e$ and define the spherical sub-algebra to be $e \mathcal{H}_{d} e$. We will prove all results in the first case, namely $\mathcal{H}_{V}$ and its spherical sub-algebra. The proofs in all other cases follow the same principles and we omit them for brevity.

Throughout this section we assume $q^{m} \neq 1$ for all $m \in \mathbb{Z}$.

\subsection{Spherical sub-algebra of $\mathcal{H}_{V}$ and PV monodromy manifold.}

Proposition 4.1. The following three elements:

$$
\begin{gathered}
X_{1}=X+W, \quad X_{2}=i \sqrt{a b}\left(T_{0}+1\right) T_{1}^{-1}-i \sqrt{\frac{1}{a b}} T_{1} T_{0}, \\
X_{3}=-i \sqrt{a b q}\left(\frac{1}{a b} W T_{0} T_{1}+\frac{1}{a b} X T_{1} T_{0}+\left(\frac{1}{a}+\frac{1}{b}\right) T_{0}+\frac{c}{a b q} T_{1}\right),
\end{gathered}
$$

commute with $e=\frac{1+T_{1}}{1-a b}$, satisfy the following quantum commutation relations:

$$
\begin{aligned}
& q^{\frac{1}{2}} X_{2} X_{1}-q^{-\frac{1}{2}} X_{1} X_{2}=\left(q-\frac{1}{q}\right) X_{3}+ \\
&+\left(q^{\frac{1}{2}}-q^{-\frac{1}{2}}\right)\left(\bar{k}_{1}+\frac{c}{\sqrt{q}}\left(\frac{i}{\sqrt{q a b}} T_{1}+i \sqrt{q a b} T_{1}^{-1}\right)\right), \\
& q^{\frac{1}{2}} X_{3} X_{2}-q^{-\frac{1}{2}} X_{2} X_{3}=\left(q^{\frac{1}{2}}-q^{-\frac{1}{2}}\right) \frac{c}{\sqrt{q}}, \\
& q^{\frac{1}{2}} X_{1} X_{3}-q^{-\frac{1}{2}} X_{3} X_{1}=\left(q-\frac{1}{q}\right) X_{2}- \\
&-\left(q^{\frac{1}{2}}-q^{-\frac{1}{2}}\right)\left(\bar{k}_{1} \frac{c}{\sqrt{q}}+\left(\frac{i}{\sqrt{q a b}} T_{1}+i \sqrt{q a b} T_{1}^{-1}\right)\right),
\end{aligned}
$$

and the quantum cubic relation:

$$
\begin{aligned}
& \qquad q^{\frac{1}{2}} X_{2} X_{1} X_{3}-q X_{2}^{2}-q X_{3}^{2}+\sqrt{q}\left(-\bar{k}_{1}-\frac{c}{\sqrt{q}}\left(\frac{i}{\sqrt{q a b}} T_{1}+i \sqrt{q a b} T_{1}^{-1}\right)\right) X_{3}- \\
& -\frac{c}{q} X_{1}+\sqrt{q}\left(\bar{k}_{1} \frac{c}{\sqrt{q}}+\left(\frac{i}{\sqrt{q a b}} T_{1}+i \sqrt{q a b} T_{1}^{-1}\right)\right) X_{2}+ \\
& \quad+1+\frac{c^{2}}{q}-\frac{c}{\sqrt{q}} \bar{k}_{1}\left(\frac{i}{\sqrt{q a b}} T_{1}+i \sqrt{q a b} T_{1}^{-1}\right)=0, \\
& \text { where } \bar{k}_{1}=i \sqrt{\frac{b}{a}}+i \sqrt{\frac{a}{b}} .
\end{aligned}
$$

Proof. To prove the quantum commutation relations and the cubic relation we use the following substitutions which can be easily be derived from $(1.7,1.8,1.9)$ and (1.10):

$$
\begin{array}{r}
T_{1}^{2}=-(a b+1) T_{1}-a b, \quad T_{0}^{2}=-T_{0} \\
T_{1} X=W T_{1}+(1+a b) W-(a+b), \\
T_{1} W=X T_{1}-(1+a b) W+a+b, \\
T_{0} X=q W T_{0}+c-X, \\
T_{0} W=\frac{1}{q} X T_{0}+\frac{1}{q} X-\frac{c}{q} .
\end{array}
$$

Using these relations recursively, it is a straightforward computation to eliminate all higher powers in $T_{0}$ and $T_{1}$ and to bring all $X$ and $W$ to the left and eventually obtain the statement. 
Corollary 4.2. In the limit $q \rightarrow 1$ the elements $X_{1}, X_{2}, X_{3}$ belong to the centre of $\mathcal{H}_{V}$ and the cubic relation (4.118) tends to the PV monodromy manifold (see Appendix A):

$X_{1} X_{2} X_{3}-X_{2}^{2}-X_{3}^{2}-c X_{1}-\left(\bar{u}_{1}-c \bar{k}_{1}\right) X_{2}-\left(-c \bar{u}_{1}+\bar{k}_{1}\right) X_{3}+1+c^{2}+c \bar{k}_{1} \bar{u}_{1}=0$.

Proof. Observe that in the limit $q \rightarrow 1$,

$$
\frac{i}{\sqrt{q a b}} T_{1}+i \sqrt{q a b} T_{1}^{-1} \rightarrow-\frac{i(1+a b)}{\sqrt{a b}}=-i \bar{u}_{1},
$$

so that we obtain (4.120) by substituting $q=1$ and $\frac{i}{\sqrt{q a b}} T_{1}+i \sqrt{q a b} T_{1}^{-1} \rightarrow-\frac{i(1+a b)}{\sqrt{a b}}$ by $-i \bar{u}_{1}$ in (4.118).

Corollary 4.3. Let $e=\frac{1+T_{1}}{1-a b}$, then the elements $\hat{X}_{i}=e X_{i} e, i=1,2,3$, where $X_{1}, X_{2}, X_{3}$ are defined by (4.116), generate the spherical sub-algebra $e \mathcal{H}_{V} e$, they satisfy the quantum commutation relations:

$$
\begin{aligned}
& q^{\frac{1}{2}} \hat{X}_{2} \hat{X}_{1}-q^{-\frac{1}{2}} \hat{X}_{1} \hat{X}_{2}=\left(q-\frac{1}{q}\right) \hat{X}_{3}-\left(q^{\frac{1}{2}}-q^{-\frac{1}{2}}\right) \omega_{3} e \\
& q^{\frac{1}{2}} \hat{X}_{3} \hat{X}_{2}-q^{-\frac{1}{2}} \hat{X}_{2} \hat{X}_{3}=-\left(q^{\frac{1}{2}}-q^{-\frac{1}{2}}\right) \omega_{1} e \\
& q^{\frac{1}{2}} \hat{X}_{1} \hat{X}_{3}-q^{-\frac{1}{2}} \hat{X}_{3} \hat{X}_{1}=\left(q-\frac{1}{q}\right) \hat{X}_{2}-\left(q^{\frac{1}{2}}-q^{-\frac{1}{2}}\right) \omega_{2} e
\end{aligned}
$$

and the quantum cubic relation:

$$
q^{\frac{1}{2}} \hat{X}_{2} \hat{X}_{1} \hat{X}_{3}-q \hat{X}_{2}^{2}-q \hat{X}_{3}^{2}+\sqrt{q} \omega_{2} \hat{X}_{2}+\frac{1}{\sqrt{q}} \omega_{1} \hat{X}_{1}+\sqrt{q} \omega_{3} \hat{X}_{3}-\omega_{4} e=0
$$

where

$$
\begin{aligned}
& \omega_{1}=-\frac{c}{\sqrt{q}}, \quad \omega_{2}=\frac{c}{\sqrt{q}} \bar{k}_{1}-i\left(\sqrt{\frac{a b}{q}}+\sqrt{\frac{q}{a b}}\right), \\
& \omega_{3}=-\bar{k}_{1}+i \frac{c}{\sqrt{q}}\left(\sqrt{\frac{a b}{q}}+\sqrt{\frac{q}{a b}}\right), \\
& \omega_{4}=1+\frac{c^{2}}{q}+i \frac{c \bar{k}_{1}}{\sqrt{q}}\left(\sqrt{\frac{a b}{q}}+\sqrt{\frac{q}{a b}}\right) .
\end{aligned}
$$

Proof. The fact that $\hat{X}_{1}, \hat{X}_{2}, \hat{X}_{3}$ and $e$ generate the spherical sub-algebra $e \mathcal{H}_{V}$ follows easily from the following relations

$$
e T_{0} e=\frac{i \sqrt{a b}}{1-a b} \hat{X}_{2}-\frac{1}{1-a b} e, \quad e T_{1} e=-a b e, \quad e X e=\frac{1}{1-a b} \hat{X}_{1}-\frac{a+b}{1-a b} e
$$

which can be proved as in the proof of Proposition 4.1.

To prove the quantum commutation relations (4.121) it is enough to observe that $e$ is idempotent and to prove that $X_{1}, X_{2}, X_{3}$ commute with $e$. Indeed if this is true, we can just multiply (4.117) by $e$ and use the fact that $e X_{i} X_{j}=e^{2} X_{i} X_{j}=$ $e X_{i} e X_{j}=\hat{X}_{i} \hat{X}_{j}$. In a similar way we can prove (4.122) by multiplying (4.118) by $e$ three times.

So we only need to prove that $\left[e, X_{1,2}\right]=0$ :

$$
(1-a b)\left[e, X_{1}\right]=\left[1+T_{1}, X+W\right]=T_{1} X+T_{1} W-X T_{1}-W T_{1}=
$$




$$
\begin{aligned}
& =\left(W T_{1}+(1+a b) W-(a+b)\right)+\left(X T_{1}-(1+a b) W+a+b\right)-X T_{1}-W T_{1}=0 . \\
& (1-a b)\left[e, X_{2}\right]=\left[1+T_{1}, i \sqrt{a b}\left(T_{0}+1\right) T_{1}^{-1}-i \sqrt{\frac{1}{a b}} T_{1} T_{0}\right]= \\
& =i \sqrt{a b} T_{1}\left(T_{0}+1\right) T_{1}^{-1}-i \sqrt{\frac{1}{a b}} T_{1}^{2} T_{0}-i \sqrt{a b} T_{0}\left(T_{0}+1\right)-i \sqrt{\frac{1}{a b}} T_{0} T_{1} T_{0}= \\
& =-i \sqrt{a b} T_{1}\left(T_{0}+1\right)\left(\frac{1}{a b} T_{1}+\frac{1+a b}{a b}\right)-i \sqrt{\frac{1}{a b}}\left(-(a b+1) T_{1}-a b\right) T_{0}-i \sqrt{\frac{1}{a b}} T_{0} T_{1} T_{0}=0 .
\end{aligned}
$$

This concludes the proof.

\subsection{Spherical sub-algebra of $\mathcal{H}_{I V}$ and PIV monodromy manifold.}

Proposition 4.4. The following three elements:

$$
\begin{gathered}
X_{1}=X+W, \quad X_{2}=i \sqrt{a b}\left(T_{0}+1\right) T_{1}^{-1}-i \sqrt{\frac{1}{a b}} T_{1} T_{0}, \\
X_{3}=-i \sqrt{\frac{q}{a b}}\left(W T_{0} T_{1}+X T_{1} T_{0}-i \sqrt{a b} T_{0}+\frac{c}{q} T_{1}\right)
\end{gathered}
$$

commute with $e=\frac{1+T_{1}}{1-a b}$, satisfy the following quantum commutation relations:

$$
\begin{aligned}
& q^{\frac{1}{2}} X_{2} X_{1}-q^{-\frac{1}{2}} X_{1} X_{2}=\left(q-\frac{1}{q}\right) X_{3}+ \\
&+\left(q^{\frac{1}{2}}-q^{-\frac{1}{2}}\right)\left(-1+\frac{c}{\sqrt{q}}\left(\frac{i}{\sqrt{q a b}} T_{1}+i \sqrt{q a b} T_{1}^{-1}\right)\right) \\
& q^{\frac{1}{2}} X_{3} X_{2}-q^{-\frac{1}{2}} X_{2} X_{3}=\left(q^{\frac{1}{2}}-q^{-\frac{1}{2}}\right) \frac{c}{\sqrt{q}} \\
& q^{\frac{1}{2}} X_{1} X_{3}-q^{-\frac{1}{2}} X_{3} X_{1}=\left(q^{\frac{1}{2}}-q^{-\frac{1}{2}}\right) \frac{c}{\sqrt{q}}
\end{aligned}
$$

and the quantum cubic relation:

$$
\begin{gathered}
q^{\frac{1}{2}} X_{2} X_{1} X_{3}-q X_{3}^{2}-c X_{2}-\frac{c}{q} X_{1}+\frac{c^{2}}{q}+\frac{c}{\sqrt{q}}\left(\frac{i}{\sqrt{q a b}} T_{1}+i \sqrt{q a b} T_{1}^{-1}\right)+ \\
+\sqrt{q}\left(1-\frac{c}{\sqrt{q}}\left(\frac{i}{\sqrt{q a b}} T_{1}+i \sqrt{q a b} T_{1}^{-1}\right)\right) X_{3}
\end{gathered}
$$

Corollary 4.5. In the limit $q \rightarrow 1$ the elements $X_{1}, X_{2}, X_{3}$ belong to the centre of $\mathcal{H}_{I V}$ and the cubic relation (4.126) tends to the PIV monodromy manifold (see Appendix A):

$$
X_{1} X_{2} X_{3}-X_{3}^{2}-c X_{1}-c X_{2}+\left(1+\frac{c}{b}-c b\right) X_{3}+c\left(c+b-\frac{1}{b}\right)=0 .
$$

Corollary 4.6. The elements $\hat{X}_{i}=e X_{i} e, i=1,2,3$, where $X_{1}, X_{2}, X_{3}$ are defined by (4.124), generate the spherical sub-algebra $e \mathcal{H} e$, satisfy the quantum commutation relations:

$$
\begin{aligned}
& q^{\frac{1}{2}} \hat{X}_{2} \hat{X}_{1}-q^{-\frac{1}{2}} \hat{X}_{1} \hat{X}_{2}=\left(q-\frac{1}{q}\right) \hat{X}_{3}-\left(q^{\frac{1}{2}}-q^{-\frac{1}{2}}\right) \omega_{3} e \\
& q^{\frac{1}{2}} \hat{X}_{3} \hat{X}_{2}-q^{-\frac{1}{2}} \hat{X}_{2} \hat{X}_{3}=-\left(q^{\frac{1}{2}}-q^{-\frac{1}{2}}\right) \omega_{1} e \\
& q^{\frac{1}{2}} \hat{X}_{1} \hat{X}_{3}-q^{-\frac{1}{2}} \hat{X}_{3} \hat{X}_{1}=-\left(q^{\frac{1}{2}}-q^{-\frac{1}{2}}\right) \omega_{2} e
\end{aligned}
$$


and the quantum cubic relation

$$
q^{\frac{1}{2}} \hat{X}_{2} \hat{X}_{1} \hat{X}_{3}-q \hat{X}_{3}^{2}+\sqrt{q} \omega_{2} \hat{X}_{2}+\frac{1}{\sqrt{q}} \omega_{1} \hat{X}_{1}+\sqrt{q} \omega_{3} \hat{X}_{3}+\omega_{4} e=0,
$$

where

$\omega_{1}=\omega_{2}=-\frac{c}{\sqrt{q}}, \quad \omega_{3}=1+\frac{c}{\sqrt{q}} i\left(\sqrt{\frac{a b}{q}}+\sqrt{\frac{q}{a b}}\right), \quad \omega_{4}=\frac{c^{2}}{q}-\frac{c}{\sqrt{q}} i\left(\sqrt{\frac{a b}{q}}+\sqrt{\frac{q}{a b}}\right)$.

\subsection{Spherical sub-algebra of $\mathcal{H}_{I I I}$ and PIII monodromy manifold.}

Proposition 4.7. The following three elements:

$$
\begin{gathered}
X_{1}=X+W, \quad X_{2}=i \sqrt{a b} T_{0} T_{1}^{-1}-i \sqrt{\frac{1}{a b}} T_{1} T_{0}, \\
X_{3}=-i \sqrt{a b q}\left(\frac{1}{a b} W T_{0} T_{1}+\frac{1}{a b} X T_{1} T_{0}+\left(\frac{1}{a}+\frac{1}{b}\right) T_{0}-\frac{1}{a b q} T_{1}\right),
\end{gathered}
$$

commute with $e=\frac{1+T_{1}}{1-a b}$, satisfy the following quantum commutation relations:

$$
\begin{aligned}
& q^{\frac{1}{2}} X_{2} X_{1}-q^{-\frac{1}{2}} X_{1} X_{2}=\left(q-\frac{1}{q}\right) X_{3}-q^{-\frac{1}{2}}\left(q^{\frac{1}{2}}-q^{-\frac{1}{2}}\right)\left(\frac{i}{\sqrt{q a b}} T_{1}+i \sqrt{q a b} T_{1}^{-1}\right), \\
& q^{\frac{1}{2}} X_{3} X_{2}-q^{-\frac{1}{2}} X_{2} X_{3}=0 \\
& q^{\frac{1}{2}} X_{1} X_{3}-q^{-\frac{1}{2}} X_{3} X_{1}=\left(q-\frac{1}{q}\right) X_{2}+\left(q^{\frac{1}{2}}-q^{-\frac{1}{2}}\right) q^{-\frac{1}{2}} \bar{k}_{1},
\end{aligned}
$$

and the quantum cubic relation:

(4.133) $q^{\frac{1}{2}} X_{2} X_{1} X_{3}-q X_{2}^{2}-q X_{3}^{2}-\bar{k}_{1} X_{2}+\left(\frac{i}{\sqrt{q a b}} T_{1}+i \sqrt{q a b} T_{1}^{-1}\right) X_{3}+1 / q=0$,

where $\bar{k}_{1}=i \sqrt{\frac{b}{a}}+i \sqrt{\frac{a}{b}}$.

Corollary 4.8. In the limit $q \rightarrow 1$ the elements $X_{1}, X_{2}, X_{3}$ generate the centre of $\mathcal{H}_{I I I}$ and the cubic relation (4.133) tends to the PIII monodromy manifold (see Appendix A):

$$
X_{1} X_{2} X_{3}-X_{2}^{2}-X_{3}^{2}-\left(i \sqrt{\frac{b}{a}}+i \sqrt{\frac{a}{b}}\right) X_{2}-\left(i \sqrt{a b}+i \frac{1}{\sqrt{a b}}\right) X_{3}+1=0 .
$$

Corollary 4.9. The elements $\hat{X}_{i}=e X_{i} e, i=1,2,3$, where $X_{1}, X_{2}, X_{3}$ are defined by (4.131), generate the spherical sub-algebra $e \mathcal{H} e$, they satisfy the quantum commutation relations:

$$
\begin{aligned}
& q^{\frac{1}{2}} \hat{X}_{2} \hat{X}_{1}-q^{-\frac{1}{2}} \hat{X}_{1} \hat{X}_{2}=\left(q-\frac{1}{q}\right) \hat{X}_{3}-\left(q^{\frac{1}{2}}-q^{-\frac{1}{2}}\right) \omega_{3} e, \\
& q^{\frac{1}{2}} \hat{X}_{3} \hat{X}_{2}-q^{-\frac{1}{2}} \hat{X}_{2} \hat{X}_{3}=0, \\
& q^{\frac{1}{2}} \hat{X}_{1} \hat{X}_{3}-q^{-\frac{1}{2}} \hat{X}_{3} \hat{X}_{1}=\left(q-\frac{1}{q}\right) \hat{X}_{2}-\left(q^{\frac{1}{2}}-q^{-\frac{1}{2}}\right) \omega_{2} e,
\end{aligned}
$$

and the quantum cubic relation:

$$
q^{\frac{1}{2}} \hat{X}_{2} \hat{X}_{1} \hat{X}_{3}-q \hat{X}_{2}^{2}-q \hat{X}_{3}^{2}+\sqrt{q} \omega_{2} \hat{X}_{2}+\sqrt{q} \omega_{3} \hat{X}_{3}+e=0,
$$


where

$$
\omega_{2}=-\frac{i}{\sqrt{q}}\left(\sqrt{\frac{b}{a}}-i \sqrt{\frac{a}{b}}\right), \quad \omega_{3}=-\frac{i}{\sqrt{q}}\left(\sqrt{\frac{a b}{q}}+\sqrt{\frac{q}{a b}}\right) .
$$

4.4. Spherical sub-algebra of $\mathcal{H}_{I I I^{D_{7}}}$ and $P I I I^{D_{7}}$ monodromy manifold.

Lemma 4.10. The generators $X, W, T_{0}, T_{1}$ of the confluent Cherednik algebra $\mathcal{H}_{I I I}^{D_{7}}$ satisfy the following relations

$$
\begin{array}{ll}
T_{1} X T_{1}=-a T_{1}, & \left(T_{1}+1\right) W\left(T_{1}+1\right)=a\left(T_{1}+1\right), \\
T_{0} W T_{0}=-\frac{1}{q} T_{0}, & T_{0} X T_{0}=T_{0} .
\end{array}
$$

Proposition 4.11. The following three elements:

$$
X_{1}=X+W
$$

$$
X_{3}=\frac{q}{q^{2}-1}\left(q^{1 / 2} X_{2} X_{1}-q^{-1 / 2} X_{1} X_{2}\right)-\frac{1}{q+1}\left(\left(\sqrt{q}-\frac{1}{\sqrt{q}}\right) T_{1}+\sqrt{q}\right),
$$

commute with $e=1+T_{1}$, they satisfy the quantum commutation relations:

(4.140) $q^{\frac{1}{2}} X_{3} X_{2}-q^{-\frac{1}{2}} X_{2} X_{3}=0$,

$$
q^{\frac{1}{2}} X_{2} X_{1}-q^{-\frac{1}{2}} X_{1} X_{2}=\left(q-\frac{1}{q}\right) X_{3}+\frac{q-1}{q}\left(\left(\sqrt{q}-\frac{1}{\sqrt{q}}\right) T_{1}+\sqrt{q}\right),
$$

$$
q^{\frac{1}{2}} X_{1} X_{3}-q^{-\frac{1}{2}} X_{3} X_{1}=\left(q-\frac{1}{q}\right) X_{2}-\frac{q-1}{q} a,
$$

and the quantum cubic relation:

$$
q^{\frac{1}{2}} X_{2} X_{1} X_{3}-q X_{2}^{2}-q X_{3}^{2}+a X_{2}+\left(q^{-\frac{1}{2}} T_{1}-q^{\frac{1}{2}}\left(T_{1}+1\right)\right) X_{3}=0 .
$$

Corollary 4.12. In the limit $q \rightarrow 1$, the elements $X_{1}, X_{2}, X_{3}$ become central and the cubic relation (4.141) tends to the the $P I I I^{D_{7}}$ monodromy manifold (see Appendix A):

$$
X_{3} X_{2} X_{1}-X_{2}^{2}-X_{3}^{2}-a X_{2}+X_{3}=0 .
$$

Corollary 4.13. Define $\hat{X}_{i}=e X_{i} e, i=1,2,3$, where $X_{1}, X_{2}, X_{3}$ are defined by (4.139) and

$$
e=1+T_{1} \text {. }
$$

Then $\hat{X}_{1}, \hat{X}_{2}, \hat{X}_{3}$ generate the spherical sub-algebra $e \mathcal{H}_{I I I}^{D_{7}} e$, they satisfy the quantum commutation relations:

$$
\begin{aligned}
& q^{\frac{1}{2}} \hat{X}_{2} \hat{X}_{1}-q^{-\frac{1}{2}} \hat{X}_{1} \hat{X}_{2}=\left(q-\frac{1}{q}\right) \hat{X}_{3}-\left(q^{\frac{1}{2}}-q^{-\frac{1}{2}}\right) e \\
& q^{\frac{1}{2}} \hat{X}_{3} \hat{X}_{2}-q^{-\frac{1}{2}} \hat{X}_{2} \hat{X}_{3}=0 \\
& q^{\frac{1}{2}} \hat{X}_{1} \hat{X}_{3}-q^{-\frac{1}{2}} \hat{X}_{3} \hat{X}_{1}=\left(q-\frac{1}{q}\right) \hat{X}_{2}-\left(q^{\frac{1}{2}}-q^{-\frac{1}{2}}\right) \frac{a}{\sqrt{q}} e
\end{aligned}
$$

and the quantum cubic cubic relation:

$$
q^{\frac{1}{2}} \hat{X}_{2} \hat{X}_{1} \hat{X}_{3}-q \hat{X}_{2}^{2}-q \hat{X}_{3}^{2}+a \hat{X}_{2}-\frac{1}{\sqrt{q}} \hat{X}_{3}=0 .
$$


4.5. Spherical sub-algebra of $\mathcal{H}_{I I I^{D_{8}}}$ and $P I I I^{D_{8}}$ monodromy manifold. Here all proofs are a simple limit as $a \rightarrow 0$ of the proofs of the previous Sub-section and will be omitted.

Lemma 4.14. The generators $X, W, T_{0}, T_{1}$ of the confluent Cherednik algebra $\mathcal{H}_{I I I^{D_{8}}}$ satisfy the following relations

$$
\begin{aligned}
& T_{1} X T_{1}=0, \quad\left(T_{1}+1\right) W\left(T_{1}+1\right)=0, \\
& T_{0} W T_{0}=-\frac{1}{q} T_{0}, \quad T_{0} X T_{0}=T_{0} .
\end{aligned}
$$

Proposition 4.15. The following three elements:

$$
\begin{array}{r}
X_{1}=X+W \\
X_{2}=T_{1} T_{0}+T_{0}\left(T_{1}+1\right) \\
X_{3}=\frac{q}{q^{2}-1}\left(q^{1 / 2} X_{2} X_{1}-q^{-1 / 2} X_{1} X_{2}\right)-\frac{1}{q+1}\left(\left(\sqrt{q}-\frac{1}{\sqrt{q}}\right) T_{1}+\sqrt{q}\right),
\end{array}
$$

commute with $e=1+T_{1}$, satisfy the following quantum commutation relations:

(4.148) $q^{\frac{1}{2}} X_{3} X_{2}-q^{-\frac{1}{2}} X_{2} X_{3}=0$,

$$
q^{\frac{1}{2}} X_{2} X_{1}-q^{-\frac{1}{2}} X_{1} X_{2}=\left(q-\frac{1}{q}\right) X_{3}+\frac{q-1}{q}\left(\left(\sqrt{q}-\frac{1}{\sqrt{q}}\right) T_{1}+\sqrt{q}\right),
$$

$$
q^{\frac{1}{2}} X_{1} X_{3}-q^{-\frac{1}{2}} X_{3} X_{1}=\left(q-\frac{1}{q}\right) X_{2},
$$

and the quantum cubic relation:

$$
q^{\frac{1}{2}} X_{2} X_{1} X_{3}-q X_{2}^{2}-q X_{3}^{2}+\left(q^{-\frac{1}{2}} T_{1}-q^{\frac{1}{2}}\left(T_{1}+1\right)\right) X_{3}=0 .
$$

Corollary 4.16. In the limit $q \rightarrow 1$, the elements $X_{1}, X_{2}, X_{3}$ become central and the cubic relation (4.149) tends to the the $P I I I^{D_{8}}$ monodromy manifold (see Appendix A):

$$
X_{3} X_{2} X_{1}-X_{2}^{2}-X_{3}^{2}+X_{3}=0 \text {. }
$$

Corollary 4.17. Define $\hat{X}_{i}=e X_{i} e, i=1,2$, where $X_{1}, X_{2}, X_{3}$ are defined by (4.147) and

$$
e=1+T_{1} \text {. }
$$

Then $\hat{X}_{1}, \hat{X}_{2}$ generate the spherical sub-algebra $e \mathcal{H}_{I I I^{D_{8}}} e$, the satisfy the quantum commutation relations:

$$
\begin{aligned}
& q^{\frac{1}{2}} \hat{X}_{2} \hat{X}_{1}-q^{-\frac{1}{2}} \hat{X}_{1} \hat{X}_{2}=\left(q-\frac{1}{q}\right) \hat{X}_{3}-\frac{q-1}{\sqrt{q}} e \\
& q^{\frac{1}{2}} \hat{X}_{3} \hat{X}_{2}-q^{-\frac{1}{2}} \hat{X}_{2} \hat{X}_{3}=0 \\
& q^{\frac{1}{2}} \hat{X}_{1} \hat{X}_{3}-q^{-\frac{1}{2}} \hat{X}_{3} \hat{X}_{1}=\left(q-\frac{1}{q}\right) \hat{X}_{2}
\end{aligned}
$$

and lie on the following quantum cubic

$$
q^{\frac{1}{2}} \hat{X}_{2} \hat{X}_{1} \hat{X}_{3}-q \hat{X}_{2}^{2}-q \hat{X}_{3}^{2}-\frac{1}{\sqrt{q}} \hat{X}_{3}=0 .
$$




\subsection{Spherical sub-algebra of $\mathcal{H}_{I I}$ and PII monodromy manifold.}

Proposition 4.18. The following three elements:

$$
\begin{gathered}
X_{1}=X+W, \quad X_{2}=-b\left(T_{0}+1\right) T_{1}^{-1}-\frac{1}{b} T_{1} T_{0}, \\
X_{3}=-\frac{\sqrt{q}}{b}\left(W T_{0} T_{1}+X T_{1} T_{0}-\frac{1}{\sqrt{q}} T_{1}\right)
\end{gathered}
$$

commute with $e=\frac{1+T_{1}}{1+b^{2}}$, satisfy the following quantum commutation relations:

$$
\begin{aligned}
& q^{\frac{1}{2}} X_{2} X_{1}-q^{-\frac{1}{2}} X_{1} X_{2}=\left(q-\frac{1}{q}\right) X_{3}+\left(q^{\frac{1}{2}}-q^{-\frac{1}{2}}\right)\left(-\frac{1}{\sqrt{q} b} T_{1}+\sqrt{q} b T_{1}^{-1}\right), \\
& q^{\frac{1}{2}} X_{3} X_{2}-q^{-\frac{1}{2}} X_{2} X_{3}=-\left(q^{\frac{1}{2}}-q^{-\frac{1}{2}}\right), \\
& q^{\frac{1}{2}} X_{1} X_{3}-q^{-\frac{1}{2}} X_{3} X_{1}=0
\end{aligned}
$$

and the quantum cubic relation:

$$
q^{\frac{1}{2}} X_{2} X_{1} X_{3}-q X_{3}^{2}+\frac{1}{\sqrt{q}} X_{1}+\sqrt{q}\left(-\frac{1}{\sqrt{q} b} T_{1}+\sqrt{q} b T_{1}^{-1}\right) X_{3}+1=0
$$

Corollary 4.19. In the limit $q \rightarrow 1$ the elements $X_{1}, X_{2}, X_{3}$ belong to the centre of $\mathcal{H}_{I I}$ and the cubic relation (4.155) tends to the PII monodromy manifold (see Appendix A):

$$
X_{1} X_{2} X_{3}-X_{3}^{2}+X_{1}+\left(b-\frac{1}{b}\right) X_{3}+1=0 .
$$

Corollary 4.20. The elements $\hat{X}_{i}=e X_{i} e, i=1,2,3$, where $X_{1}, X_{2}, X_{3}$ are defined by (4.153), generate the spherical sub-algebra $e \mathcal{H}_{I I} e$, satisfy the quantum commutation relations:

$$
\begin{aligned}
& q^{\frac{1}{2}} \hat{X}_{2} \hat{X}_{1}-q^{-\frac{1}{2}} \hat{X}_{1} \hat{X}_{2}=\left(q-\frac{1}{q}\right) \hat{X}_{3}-\left(q^{\frac{1}{2}}-q^{-\frac{1}{2}}\right) \omega_{3} e, \\
& q^{\frac{1}{2}} \hat{X}_{3} \hat{X}_{2}-q^{-\frac{1}{2}} \hat{X}_{2} \hat{X}_{3}=-\left(q^{\frac{1}{2}}-q^{-\frac{1}{2}}\right) e, \\
& q^{\frac{1}{2}} \hat{X}_{1} \hat{X}_{3}-q^{-\frac{1}{2}} \hat{X}_{3} \hat{X}_{1}=0,
\end{aligned}
$$

and the quantum cubic relation

$$
q^{\frac{1}{2}} \hat{X}_{2} \hat{X}_{1} \hat{X}_{3}-q \hat{X}_{3}^{2}+\frac{1}{\sqrt{q}} \hat{X}_{1}+\sqrt{q} \omega_{3} \hat{X}_{3}+e=0
$$

where

$$
\omega_{3}=\left(\frac{b}{\sqrt{q}}-\frac{\sqrt{q}}{b}\right) .
$$

4.7. Spherical sub-algebra of $\mathcal{H}_{I}$ and PI monodromy manifold.

Proposition 4.21. The following three elements:

$$
\begin{array}{r}
X_{1}=\check{V}_{1} V_{1}+V_{1}\left(\check{V}_{1}+1\right), \\
X_{2}=\check{V}_{1} V_{0}+\left(V_{0}+1\right)\left(\check{V}_{1}+1\right) \\
X_{3}=q^{1 / 2} V_{1} V_{0}+q^{-1 / 2}\left(V_{0}+1\right) V_{1},
\end{array}
$$

commute with $e=1+\check{V}_{1}$, satisfy the following quantum commutation relations:

$$
q^{\frac{1}{2}} X_{2} X_{1}-q^{-\frac{1}{2}} X_{1} X_{2}=\left(q^{\frac{1}{2}}-q^{-\frac{1}{2}}\right)\left(q^{-\frac{1}{2}} \check{V}_{1}-q^{\frac{1}{2}}\left(\check{V}_{1}+1\right)\right),
$$




$$
\begin{aligned}
& q^{\frac{1}{2}} X_{3} X_{2}-q^{-\frac{1}{2}} X_{2} X_{3}=-\left(q^{\frac{1}{2}}-q^{-\frac{1}{2}}\right), \\
& q^{\frac{1}{2}} X_{1} X_{3}-q^{-\frac{1}{2}} X_{3} X_{1}=0
\end{aligned}
$$

and the quantum cubic relation:

$$
q^{\frac{1}{2}} X_{2} X_{1} X_{3}+\frac{1}{\sqrt{q}} X_{1}-\sqrt{q}\left(q^{-\frac{1}{2}} \check{V}_{1}-q^{\frac{1}{2}}\left(\check{V}_{1}+1\right)\right) X_{3}+1=0 .
$$

Corollary 4.22. In the limit $q \rightarrow 1$ the elements $X_{1}, X_{2}, X_{3}$ belong to the centre of $\mathcal{H}_{I}$ and the cubic relation (4.161) tends to the PI monodromy manifold (see Appendix A):

$$
X_{1} X_{2} X_{3}+X_{1}+X_{3}+1=0 .
$$

Corollary 4.23. The elements $\hat{X}_{i}=e X_{i} e, i=1,2,3$, where $X_{1}, X_{2}, X_{3}$ are defined by (4.160), generate the spherical sub-algebra $e \mathcal{H}_{I} e$, satisfy the quantum commutation relations:

$$
\begin{aligned}
& q^{\frac{1}{2}} \hat{X}_{2} \hat{X}_{1}-q^{-\frac{1}{2}} \hat{X}_{1} \hat{X}_{2}=-\left(q^{\frac{1}{2}}-q^{-\frac{1}{2}}\right) \sqrt{q} e \\
& q^{\frac{1}{2}} \hat{X}_{3} \hat{X}_{2}-q^{-\frac{1}{2}} \hat{X}_{2} \hat{X}_{3}=-\left(q^{\frac{1}{2}}-q^{-\frac{1}{2}}\right) e \\
& q^{\frac{1}{2}} \hat{X}_{1} \hat{X}_{3}-q^{-\frac{1}{2}} \hat{X}_{3} \hat{X}_{1}=0
\end{aligned}
$$

and the quantum cubic relation

$$
q^{\frac{1}{2}} \hat{X}_{2} \hat{X}_{1} \hat{X}_{3}+\frac{1}{\sqrt{q}} \hat{X}_{1}+q \hat{X}_{3}+e=0 .
$$

5. Embedding of the (COnfluent) Cherednik algebras into $\operatorname{Mat}\left(2, \mathbb{T}_{q}\right)$

In this section we give an embedding for $\mathcal{H}, \mathcal{H}_{V}, \mathcal{H}_{I V}, \mathcal{H}_{I I I}$ and $\mathcal{H}_{I I}$ into $\operatorname{Mat}\left(2, \mathbb{T}_{q}\right)$. The proof of such embedding is based on the Lusztig-Demazure presentation given in the following:

Lemma 5.1. The algebras $\mathcal{H}, \mathcal{H}_{V}, \mathcal{H}_{I V}, \mathcal{H}_{I I I}, \mathcal{H}_{I I}$ are the algebras generated by five elements $T^{ \pm 1}, X, W, Y, Z$, where

$$
T=T_{1}, \quad Y=T_{1} T_{0},
$$

and

$$
Z=\left\{\begin{array}{lr}
\left(T_{0}+\frac{c d}{q}+1\right)\left(T_{1}+a b+1\right), & \text { for } \mathcal{H}, \\
\left(T_{0}+1\right)\left(T_{1}+a b+1\right), & \text { for } \mathcal{H}_{V}, \\
\left(T_{0}+1\right)\left(T_{1}-b^{2}+1\right), & \text { for } \mathcal{H}_{I V}, \mathcal{H}_{I I}, \\
T_{0}\left(T_{1}+a b+1\right), & \text { for } \mathcal{H}_{I I I},
\end{array}\right.
$$

satisfying the following relations respectively:

\section{- $\mathcal{H}$}

$$
\begin{aligned}
X W=W X & =1, \\
Y Z=Z Y & =1, \\
X T+a b T^{-1} W+a+b & =0, \\
Z T+\frac{q}{c d} T^{-1} Y+1+\frac{q}{c d} & =0, \\
(T+a b)(T+1) & =0,
\end{aligned}
$$




$$
Y X=-\frac{q}{a b} T^{2} X Y-q\left(\frac{1}{a}+\frac{1}{b}\right) T Y-\left(1+\frac{c d}{q}\right) T X+(c+d) T .
$$

- $\mathcal{H}_{V}$

$$
\begin{aligned}
& W X=X W=1, \\
& Z Y=Y Z=0, \\
& X T+a b T^{-1} W+a+b=0, \\
& Z T+T^{-1} Y+1=0, \\
&(T+a b)(T+1)=0, \\
& Y X=-\frac{q}{a b} T^{2} X Y-q\left(\frac{1}{a}+\frac{1}{b}\right) T Y-T X+c T .
\end{aligned}
$$

- $\mathcal{H}_{I V}$ :

- $\mathcal{H}_{I I I}$ :

$$
\begin{array}{r}
W X=X W=0, \\
Z Y=Y Z=0, \\
X T=b^{2} T^{-1} W-b, \\
Z T+T^{-1} Y+1=0, \\
\left(T-b^{2}\right)(T+1)=0, \\
Y X=\frac{q}{b^{2}} T^{2} X Y+\frac{q}{b} T Y-T X+c T .
\end{array}
$$

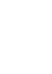

$$
\begin{aligned}
W X=X W & =1, \\
Z Y=Y Z & =0, \\
X T+a b T^{-1} W+a+b & =0, \\
Z T+T^{-1} Y & =0, \\
(T+a b)(T+1) & =0, \\
Y X=-\frac{q}{a b} T^{2} X Y-q\left(\frac{1}{a}+\frac{1}{b}\right) T Y & -T .
\end{aligned}
$$

- $\mathcal{H}_{I I}$ :

$$
\begin{array}{r}
W X=X W=0, \\
Z Y=Y Z=0, \\
X T=b^{2} T^{-1} W, \\
Z T+T^{-1} Y+1=0, \\
\left(T-b^{2}\right)(T+1)=0, \\
Y X=\frac{q}{b^{2}} T^{2} X Y-T X-\sqrt{q} T .
\end{array}
$$

Proof. For $\mathcal{H}$, see Proposition 6.6 in [35]. For the other cases, it is enough to observe that since $T$ is invertible, we can always invert relations (5.165) to define $T_{0}=$ $T^{-1} Y$. To check that the algebra relations in the $X, W, T_{0}, T_{1}$ presentation imply the ones in the $X, W, T, Y, Z$ one and vice-versa is a straightforward computation.

Theorem 5.2. The map:

$$
T \rightarrow\left(\begin{array}{cc}
0 & \sqrt{a b} e^{S_{1}} \\
-\sqrt{a b} e^{-S_{1}} & -1-a b
\end{array}\right)
$$


(5.198)

$$
\begin{aligned}
& X \rightarrow\left(\begin{array}{cc}
\left(\sqrt{\frac{a}{b}}-\sqrt{\frac{b}{a}}\right) e^{-S_{1}}+q e^{-S_{1}} e^{-S_{2}}+\frac{1}{q} e^{-S_{1}} e^{S_{2}}-\left(\sqrt{\frac{1}{a b}}+\sqrt{a b}\right) e^{S_{2}} & -q e^{S_{1}} e^{S_{2}} \\
-\left(\sqrt{\frac{a}{b}}-\sqrt{\frac{b}{a}}\right) e^{-S_{1}}-\frac{1}{q} e^{-S_{1}} e^{S_{2}}+\left(\sqrt{\frac{1}{a b}}+\sqrt{a b}\right) e^{S_{2}} & q e^{S_{1}} e^{S_{2}}
\end{array}\right), \\
& W \rightarrow\left(\left(\sqrt{\frac{a}{b}}-\sqrt{\frac{b}{a}}\right) e^{-S_{1}}+e^{-S_{1}} e^{S_{2}}-\left(\sqrt{\frac{1}{a b}}+\sqrt{a b}\right) e^{S_{2}}\right. \\
& \left.\left(\sqrt{\frac{a}{b}}-\sqrt{\frac{b}{a}}\right) e^{-S_{1}}+e^{-S_{1}} e^{-S_{2}}+e^{-S_{1}} e^{S_{2}}+\left(\sqrt{\frac{1}{a b}}+\sqrt{a b}\right) e^{S_{2}}\right), \\
& Y \rightarrow\left(\begin{array}{c}
\left(1+\frac{c d}{q}\right) \sqrt{a b} e^{S_{1}}-\sqrt{a b} c e^{-S_{2}}-\sqrt{a b} d e^{2 S_{1}} e^{S_{2}} \\
-\frac{(1+a b)(c d+q)}{q}+(1+a b) c e^{-S_{1}} e^{-S_{2}}+(1+a b) d e^{S_{1}} e^{S_{2}}+\sqrt{a b} \frac{q+c d}{q} e^{-S_{1}}-\sqrt{a b} d e^{S_{2}}
\end{array}\right. \\
& -\sqrt{a b} d e^{2 S_{1}} e^{S_{2}} \\
& \left.(1+a b) d e^{S_{1}} e^{S_{2}}-\sqrt{a b} d e^{S_{2}}\right),
\end{aligned}
$$

and

$$
\begin{array}{r}
Z \rightarrow\left(\begin{array}{c}
\left(1+\frac{1}{a b}\right) \frac{q}{c} e^{S_{1}} e^{S_{2}}-\frac{1}{\sqrt{a b} c} e^{S_{2}} \\
\frac{(1+a b)(c d+q)}{a b c d}-\left(1+\frac{1}{a b}\right)\left(\frac{q}{d} e^{-S_{1}} e^{-S_{2}}+\frac{q}{c} e^{S_{1}} e^{S_{2}}\right)-\frac{(c d+q)}{\sqrt{a b} c d} e^{-S_{1}}+\frac{1}{\sqrt{a b} c} e^{S_{2}} \\
\frac{q^{2}}{\sqrt{a b} c} e^{2 S_{1}} e^{S_{2}} \\
\frac{(c d+q)}{\sqrt{a b} c d} e^{S_{1}}-\frac{1}{\sqrt{a b} d} e^{-S_{2}}-\frac{q^{2}}{\sqrt{a b} c} e^{2 S_{1}} e^{S_{2}}
\end{array}\right),
\end{array}
$$

where $S_{1}, S_{2}$ satisfy the following commutation relations:

$$
\left[S_{1}, S_{2}\right]=i \pi \hbar
$$

and $q=e^{-i \pi \hbar}$, gives an embedding of $\mathcal{H}$ into $\operatorname{Mat}\left(2, \mathbb{T}_{q}\right)$. In particular, the images of $X, W, T, Z, T$ in $G L\left(2, \mathbb{T}_{q}\right)$ satisfy the relations $(5.167, \ldots, 5.172)$ where the quantum ordering is dictated by the matrix product ordering ${ }^{5}$.

Proof. First of all, note that since where $S_{1}, S_{2}$ satisfy the commutation relations (5.202), their exponentials satisfy the quantum torus commutation relations:

$$
e^{S_{2}} e^{S_{1}}=q e^{S_{1}} e^{S_{2}}
$$

for $q=e^{-i \pi \hbar}$ and therefore the images of $X, W, T, Z, T$ belong to $\operatorname{Mat}\left(2, \mathbb{T}_{q}\right)$. To prove that these images satisfy the relations $(5.167, \ldots, 5.172)$ in which the quantum ordering is dictated by the matrix product ordering is a straightforward computation.

To prove that the map $H \rightarrow \operatorname{Mat}\left(2, \mathbb{T}_{q}\right)$ defined by (5.197), (5.198), (5.199), $(5.200),(5.201)$ is injective we need to prove that the images of

$$
\left\{X^{m} Y^{n}\right\}_{n, m \in \mathbb{Z}} \cup\left\{T X^{m} Y^{n}\right\}_{n, m \in \mathbb{Z}},
$$

\footnotetext{
${ }^{5}$ By this we mean that the product $A B$ of two matrices $A, B$ whose entries are in $\mathbb{T}_{q}$ is computed by keeping the entries of $A$ on the left matrix of the entries of $B$.
} 
are all linearly independent. To this aim, it is straightforward to prove by induction that for any $m \geq 0, n \geq 0, X^{m} Y^{n}$ always contains terms with

$$
e^{-m S_{1}}, \ldots, e^{-S_{1}}, e^{S_{1}} \ldots, e^{(m+2 n-2) S_{1}}, e^{-(m+n) S_{2}}, \ldots e^{-S_{2}}, e^{S_{2}}, \ldots e^{(m+n-1) S_{2}},
$$

and that for any $m \geq 0, n \geq 0, T X^{m} Y^{n}$ always contains terms with $e^{-(m+1) S_{1}}, \ldots, e^{-S_{1}}, e^{S_{1}}, \ldots, e^{(m+2 n-1) S_{1}}, e^{-(m+n) S_{2}}, \ldots e^{-S_{2}}, e^{S_{2}}, \ldots, e^{(m+n-1) S_{2}}$. Since $\left\{e^{k S_{1}}, e^{m S_{2}}\right\}_{n, m \in \mathbb{Z}}$ are linearly independent, it automatically follows that the images of $\left\{X^{m} Y^{n}\right\}_{n, m \in \mathbb{Z}_{\geq 0}}$ and $\left\{T X^{m} Y^{n}\right\}_{n, m \in \mathbb{Z}_{\geq 0}}$ are all linearly independent. In a similar way it can be proved that the images of $\left\{T X^{m} Y^{n}\right\}_{n, m \in \mathbb{Z}_{\leq 0}}$ and $\left\{X^{m} Y^{n}\right\}_{n, m \in \mathbb{Z}_{\leq 0}}$ are all linearly independent.

To show that all the images of $\left\{X^{m} Y^{n}\right\}_{n, m \in \mathbb{Z}} \cup\left\{T X^{m} Y^{n}\right\}_{n, m \in \mathbb{Z}}$ are linearly independent we proceed by contradiction. Assume that there exists a finite linear combination which gives zero:

$$
\sum_{n, m} a_{n, m} X^{m} Y^{n}+\sum_{n, m} b_{n, m} T X^{m} Y^{n}=0
$$

take

$$
n_{0}=\min \left\{n \mid a_{n, m} \text { or } b_{n, m} \neq 0\right\}, \quad m_{0}=\max \left\{l \mid a n, m \text { or } b_{n, m} \neq 0\right\} .
$$

and multiply the above relation by $Y^{-n_{0}} X^{-m_{0}}$. Then we obtain a zero linear combination the set $\left\{T X^{m} Y^{n}\right\}_{n, m \in \mathbb{Z}_{\geq 0}} \cup\left\{X^{m} Y^{n}\right\}_{n, m \in \mathbb{Z}_{\geq 0}}$, which is absurd.

Theorem 5.3. The map given by the same formulae as Theorem 5.2 for $T, X$ and $W$ and by

$$
\begin{gathered}
Y \rightarrow\left(\begin{array}{cc}
\sqrt{a b} e^{S_{1}}-\sqrt{a b} c e^{-S_{2}} & 0 \\
-(1+a b)+(1+a b) c e^{-S_{1}} e^{-S_{2}}+\sqrt{a b} e^{-S_{1}} & 0
\end{array}\right) \\
Z \rightarrow\left(\begin{array}{cc}
0 & 0 \\
\frac{a b+1}{a b}-c \frac{a b+1}{a b} e^{-S_{1}} e^{-S_{2}}-\frac{1}{\sqrt{a b}} e^{-S_{1}} & \frac{1}{\sqrt{a b}} e^{S_{1}}-\frac{c}{q \sqrt{a b}} e^{-S_{2}}
\end{array}\right)
\end{gathered}
$$

where $S_{1}, S_{2}$ satisfy the commutation relations (5.202), gives an embedding of $\mathcal{H}_{V}$ into $\operatorname{Mat}\left(2, \mathbb{T}_{q}\right)$.

Proof. We can prove that the images of $\left\{T X^{m} Y^{n}\right\}_{n \in \mathbb{Z}_{>0}, m \in \mathbb{Z}} \cup\left\{X^{m} Y^{n}\right\}_{n \in \mathbb{Z}_{>0}, m \in \mathbb{Z}}$ are all linearly independent in the same way as in the proof of Theorem 5.2. To prove that the images of

$\left\{T X^{m} Y^{n}\right\}_{n \in \mathbb{Z}_{\geq 0}, m \in \mathbb{Z}} \cup\left\{X^{m} Y^{n}\right\}_{n \in \mathbb{Z}_{\geq 0}, m \in \mathbb{Z}} \cup\left\{T X^{m} Z^{n}\right\}_{n \in \mathbb{Z}_{\geq 0}, m \in \mathbb{Z}} \cup\left\{X^{m} Z^{n}\right\}_{n \in \mathbb{Z}_{\geq 0}, m \in \mathbb{Z}}$ are linearly independent, we proceed by contradiction: we assume that there exists a finite linear combination which gives zero:

$$
\sum_{n, m} a_{n, m} X^{m} Y^{n}+\sum_{n, m} b_{n, m} T X^{m} Y^{n}+\sum_{n, m} c_{n, m} X^{m} Z^{n}+\sum_{k, l} d_{n, m} T X^{m} Z^{n}=0,
$$

in which at least one $c_{n, m}$ or $d_{n, m}$ is non zero for $n>0$. Since the second column of $Y$ is identically zero, all elements in $\left\{T X^{m} Y^{n}\right\}_{n \in \mathbb{Z}_{>0}, m \in \mathbb{Z}} \cup\left\{X^{m} Y^{n}\right\}_{n \in \mathbb{Z}>0, m \in \mathbb{Z}}$ have the second column identically equal to zero. This means that the 12 and 22 elements of

$$
\sum_{m} a_{0, m} X^{m}+\sum_{m} b_{0, m} T X^{m}+\sum_{n, m} c_{n, m} X^{m} Z^{n}+\sum_{k, l} d_{n, m} T X^{m} Z^{n}
$$


must be 0 . We can prove by induction that the 12 elements of $X^{m}$ and $T X^{m}$ contain terms

$$
e^{-S_{1}}, e^{S_{1}} \ldots, e^{m S_{1}}, e^{-S_{2}}, e^{S_{2}}, \ldots e^{m S_{2}},
$$

while the 22 elements don't contain $e^{-S_{2}}$ either. The 12 and 22 elements of $X^{m} Z^{n}$ and $T X^{m} Z^{n}$ contain terms

$$
e^{-S_{1}}, e^{S_{1}} \ldots, e^{(m+n) S_{1}}, e^{-S_{2}}, e^{S_{2}}, \ldots e^{m S_{2}} .
$$

By using the fact that $\left\{e^{k S_{1}}, e^{m S_{2}}\right\}_{n, m \in \mathbb{Z}}$ are linearly independent, we conclude.

Theorem 5.4. The map:

$$
\begin{gathered}
T \rightarrow\left(\begin{array}{cc}
0 & i b e^{S_{1}} \\
-i b e^{-S_{1}} & -1+b^{2}
\end{array}\right) \\
X \rightarrow\left(\begin{array}{cc}
-i e^{-S_{1}}+\frac{1}{q} e^{-S_{1}} e^{S_{2}}-i\left(b-\frac{1}{b}\right) e^{S_{2}} & -q e^{S_{1}} e^{S_{2}} \\
i e^{-S_{1}}-\frac{1}{q} e^{-S_{1}} e^{S_{2}}+i\left(b-\frac{1}{b}\right) e^{S_{2}} & q e^{S_{1}} e^{S_{2}}
\end{array}\right)
\end{gathered}
$$

$$
W \rightarrow\left(\begin{array}{cc}
e^{S_{1}} e^{S_{2}} & e^{S_{1}} e^{S_{2}} \\
-i e^{-S_{1}}+e^{-S_{1}} e^{S_{2}}-i\left(b-\frac{1}{b}\right) e^{S_{2}} & -i e^{-S_{1}}+e^{-S_{1}} e^{S_{2}}-i\left(b-\frac{1}{b}\right) e^{S_{2}}
\end{array}\right)
$$

$$
\begin{gathered}
Y \rightarrow\left(\begin{array}{cc}
i b e^{S_{1}}-i b c e^{-S_{2}} & 0 \\
b^{2}-1+i b e^{-S_{1}}+c\left(1-b^{2}\right) e^{-S_{1}} e^{-S_{2}} & 0
\end{array}\right), \\
Z \rightarrow\left(\begin{array}{cc}
0 & 0 \\
\left(1-\frac{1}{b^{2}}\right)\left(1-c e^{-S_{1}} e^{-S_{2}}\right)+\frac{i}{b} e^{-S_{1}} & -\frac{i}{b} e^{S_{1}}+\frac{i c}{q b} e^{-S_{2}}
\end{array}\right),
\end{gathered}
$$

where $S_{1}, S_{2}$ satisfy the commutation relations (5.202), gives an embedding of $\mathcal{H}_{I V}$ into $\operatorname{Mat}\left(2, \mathbb{T}_{q}\right)$.

Proof. The proof of this Theorem is very similar to the proof of Theorem 5.3, except that in this case we need to prove that the images of

$$
\begin{aligned}
& \left\{X^{m} Y^{n}\right\}_{m, n \in \mathbb{Z}_{\geq 0}} \cup\left\{X^{m} T Y^{n}\right\}_{m, n \in \mathbb{Z}_{\geq 0}} \cup\left\{X^{m} Z^{n}\right\}_{m, n \in \mathbb{Z}_{\geq 0}} \cup\left\{X^{m} T Z^{n}\right\}_{m, n \in \mathbb{Z}_{\geq 0}} \cup \\
& \cup\left\{W^{m} Y^{n}\right\}_{m, n \in \mathbb{Z}_{\geq 0}} \cup\left\{W^{m} T Y^{n}\right\}_{m, n \in \mathbb{Z}_{\geq 0}} \cup\left\{W^{m} Z^{n}\right\}_{m, n \in \mathbb{Z}_{\geq 0}} \cup\left\{W^{m} T Z^{n}\right\}_{m, n \in \mathbb{Z}_{\geq 0}}
\end{aligned}
$$

are all linearly independent. The only novelty is that now instead of negative powers of $X$, we have positive powers of $W$.

Theorem 5.5. The map given by the same formulae as Theorem 5.2 for $T, X$ and $W$ and by

$$
\begin{gathered}
Y \rightarrow\left(\begin{array}{cc}
\sqrt{a b} e^{-S_{2}} & 0 \\
-(1+a b) e^{-S_{1}} e^{-S_{2}} & 0
\end{array}\right) \\
Z \rightarrow\left(\begin{array}{cc}
0 & 0 \\
\frac{a b+1}{a b} e^{-S_{1}} e^{-S_{2}} & \frac{1}{q \sqrt{a b}} e^{-S_{2}}
\end{array}\right)
\end{gathered}
$$

where $S_{1}, S_{2}$ satisfy the commutation relations (5.202), gives an embedding of $\mathcal{H}_{I I I}$ into $\operatorname{Mat}\left(2, \mathbb{T}_{q}\right)$.

Proof. The proof of this Theorem follows the same lines as the one of Theorem 5.3. 
Theorem 5.6. The map given by the same formulae as Theorem 5.4 for $T, Y$ and $Z$ and by

$$
\begin{aligned}
& X \rightarrow\left(\begin{array}{cc}
\frac{1}{q} e^{-S_{1}} e^{S_{2}}-i\left(b-\frac{1}{b}\right) e^{S_{2}} & -q e^{S_{1}} e^{S_{2}} \\
-\frac{1}{q} e^{-S_{1}} e^{S_{2}}+i\left(b-\frac{1}{b}\right) e^{S_{2}} & q e^{S_{1}} e^{S_{2}}
\end{array}\right) \\
& W \rightarrow\left(\begin{array}{cc}
e^{S_{1}} e^{S_{2}} & e^{S_{1}} e^{S_{2}} \\
e^{-S_{1}} e^{S_{2}}-i\left(b-\frac{1}{b}\right) e^{S_{2}} & e^{-S_{1}} e^{S_{2}}-i\left(b-\frac{1}{b}\right) e^{S_{2}}
\end{array}\right)
\end{aligned}
$$

gives an embedding of $\mathcal{H}_{I I}$ into $\operatorname{Mat}\left(2, \mathbb{T}_{q}\right)$.

Proof. The proof of this Theorem follows closely the proof of Theorem 5.4, so we omit it.

\section{Confluent Zhedanov Algebras And Q-Askey scheme}

In this Section we prove that the spherical sub-algebra of each confluent Cherednik algebra is isomorphic to the corresponding confluent Zhedanov algebra quotiented by its Casimir. Moreover we give a faithful representation of the confluent Zhedanov algebras and show that they act as symmetries of some elements of the q-Askey scheme. Throughout this section many results on basic hypergeometric polynomials are used, they can be found in [23] (see also [1] and [17] and references therein). Throughout this Section we assume $q^{m} \neq 1, \forall m \in \mathbb{Z}$.

Before stating the isomorphism theorem we need to give the Casimir element for each confluent Zhedanov algebra:

Lemma 6.1. For each index $d=V, I V, I I I, I I I^{D_{7}}, I I I^{D_{8}}, I I, I$, the Zhedanov algebra $\mathcal{Z}_{d}$ can be equivalently described as the algebra with two generators $K_{0}, K_{1}$ and two relations:

$$
\begin{aligned}
& \left(q+q^{-1}\right) K_{1} K_{0} K_{1}-K_{1}^{2} K_{0}-K_{0} K_{1}^{2}=B K_{1}+\left(q-q^{-1}\right)^{2} K_{0}+D_{0}, \\
& \left(q+q^{-1}\right) K_{0} K_{1} K_{0}-K_{0}^{2} K_{1}-K_{1} K_{0}^{2}=B K_{0}+D_{1},
\end{aligned}
$$

where the parameters $B, C_{0}, D_{0}$ and $D_{1}$ are chosen like in (1.52), and admits the following Casimir:

$$
\begin{aligned}
\mathcal{C}= & \left(K_{1} K_{0}\right)^{2}-\left(q^{2}+1+q^{-2}\right) K_{0} K_{1} K_{0} K_{1}+\left(q+q^{-1}\right)\left(q-q^{-1}\right)^{2} K_{0}^{2}+ \\
& +\left(q+q^{-1}\right) K_{0}^{2} K_{1}^{2}+B\left(\left(q+1+q^{-1}\right) K_{0} K_{1}+K_{1} K_{0}\right)+ \\
& +\left(q+1+q^{-1}\right)\left(D_{0} K_{0}+D_{1} K_{1}\right) .
\end{aligned}
$$

Let us consider the quotiented Zhedanov algebra $\mathcal{Z}_{d} /\left\langle\mathcal{C}=\mathcal{C}_{0}\right\rangle$. Then we have the following:

Theorem 6.2. For each index $d=V, I V, I I I, I I I^{D_{7}}, I I I^{D_{8}}, I I, I$ the map:

$$
i: \mathcal{Z}_{d} /\left\langle\mathcal{C}=\mathcal{C}_{0}\right\rangle \rightarrow e \mathcal{H}_{d} e
$$

defined by

$$
\begin{aligned}
& i\left(K_{0}\right):=i \sqrt{a b} \hat{X}_{2}, \quad i\left(K_{1}\right):=\hat{X}_{1}, \quad i(1):=e \\
& i\left(K_{2}\right)=i \sqrt{a b}\left(q-\frac{1}{q}\right) \hat{X}_{3}+\frac{\sqrt{q}}{1+q} B e,
\end{aligned}
$$

where the parameters $B, C_{0}, D_{0}, D_{1}$ are given in terms of the confluent Cherednik algebra parameters $a, b, c$ according to the formulae in table 1 below, is an algebra isomorphism. 


\begin{tabular}{|c||c|c|c|c|}
\hline & $B$ & $C_{0}$ & $D_{0}$ & $D_{1}$ \\
\hline $\mathcal{Z}_{V}$ & $\frac{(q-1)^{2}(c(1+a b / q)+a+b)}{q}$ & $\left(q-\frac{1}{q}\right)^{2}$ & $-\frac{(q-1)^{2}(q+1)((a+b) c+q+a b)}{q^{2}}$ & $-\frac{(q-1)^{2}(q+1) a b c}{q^{2}}$ \\
\hline $\mathcal{Z}_{I V}$ & $\frac{(q-1)^{2}\left(c\left(q-b^{2}\right)+b q\right)}{q^{2}}$ & 0 & $-\frac{(q-1)^{2}(q+1)}{q^{2}} b c$ & $\frac{(q-1)^{2}(q+1)}{q^{2}} b^{2} c$ \\
\hline $\mathcal{Z}_{I I I}$ & $-\frac{(q-1)^{2}}{q}(1+a b / q)$ & $\left(q-\frac{1}{q}\right)^{2}$ & $\frac{(q-1)^{2}(q+1)}{q^{2}}(a+b)$ & 0 \\
\hline $\mathcal{Z}_{I I I}^{D_{7}}$ & $-\frac{(q-1)^{2}}{q}$ & $\left(q-\frac{1}{q}\right)^{2}$ & $\frac{(q-1)^{2}(q+1)}{q^{2}} a$ & 0 \\
\hline $\mathcal{Z}_{I I I}^{D_{8}}$ & $-\frac{(q-1)^{2}}{q}$ & $\left(q-\frac{1}{q}\right)^{2}$ & 0 & 0 \\
\hline $\mathcal{Z}_{I I}$ & $-\frac{(q-1)^{2}}{q^{2}}\left(q-b^{2}\right) \sqrt{q}$ & 0 & 0 & $-\frac{(q-1)^{2}(q+1)}{q^{2}} b^{2} \sqrt{q}$ \\
\hline $\mathcal{Z}_{I}$ & $-\frac{(q-1)^{2}}{\sqrt{q}}$ & 0 & 0 & 0 \\
\hline
\end{tabular}

TABLE 1 . Values of the parameters $B, C_{0}, D_{0}, D_{1}$ in each confluent Zhedanov algebra in terms of the confluent Cherednik algebra parameters $a, b, c$.

Proof. To prove that $i$ is surjective it is enough to show that the relations (6.214) for each $\mathcal{Z}_{d}$ are mapped by $i$ to the corresponding quantum commutation relations for $\hat{X}_{1}, \hat{X}_{2}, \hat{X}_{3}$ in $e \mathcal{H}_{d} e$, where $d=I I, I I I, I I I^{D_{7}}, I I I^{D_{8}}, I V, V$. This is a straightforward but lengthy computation that we omit for brevity. Note that in the case of $Z_{I}\left(\mathcal{C}_{0}\right)$ the proof remains conjectural because both $a$ and $b$ are zero in that case, so that we have no way to deduce $\hat{X}_{3}$ from $K_{0}, K_{1}, K_{2}$.

To prove that the map $i$ is injective, we only need to prove that in each spherical sub-algebra $e \mathcal{H}_{d} e$, where $d=I I, I I I, I I I^{D_{7}}, I I I^{D_{8}}, I V, V$, the are no other relations a part from the quantum commutation relations for $\hat{X}_{1}, \hat{X}_{2}, \hat{X}_{3}$ and the quantum cubic relation. Let us illustrate how to proceed in the case of $e \mathcal{H}_{V} e$. We can use the cubic relation (4.122) to define $e$ in terms of $\hat{X}_{1}, \hat{X}_{2}, \hat{X}_{3}$. Then we use the quantum commutation relations (4.121) to order all words in the form $\hat{X}_{1}^{n} \hat{X}_{2}^{m} \hat{X}_{3}^{k}$. If there is a further relation, this will necessarily have the following form:

$$
\sum_{m, n, k \in \mathbb{Z}_{\geq 0}} a_{m, n, k} \hat{X}_{1}^{n} \hat{X}_{2}^{m} \hat{X}_{3}^{k}=0 .
$$

To prove that this can only be satisfied by choosing $a_{m, n, k}=0 \forall m, n, k \in \mathbb{Z}$, we use the embedding Theorem 5.3 and show by induction the image of each element $\hat{X}_{1}^{n} \hat{X}_{2}^{m} \hat{X}_{3}^{k}$ contains the following terms:

$$
e^{-(k+n+1) S_{1}}, \ldots, e^{-S_{1}}, e^{S_{1}}, \ldots, e^{(n+m+1) S_{1}}, e^{-(2 k+n+m) S_{2}}, \ldots, e^{-S_{2}}, e^{S_{2}}, \ldots, e^{n S_{2}} .
$$

Since $\left\{e^{k S_{1}}, e^{m S_{2}}\right\}_{n, m \in \mathbb{Z}}$ are linearly independent, it follows that the images of $\hat{X}_{1}^{n} \hat{X}_{2}^{m} \hat{X}_{3}^{k}$ are all linearly independent. In fact let $n_{0}=\max \left\{n: a_{m, n, k} \neq 0\right\}$. If $n_{0}>0$, then we have terms $e^{n_{0} S_{1}}$ that can't be eliminated by any other term. This proves that $n_{0}=0$. Let $m_{0}=\max \left\{m: a_{m, n, k} \neq 0\right\}$, then $m_{0}=0$ as otherwise we have terms with $e^{\left(m_{0}+1\right) S_{2}}$ that can't be eliminated by any other term. Similarly, $k_{0}=\max \left\{k: a_{m, n, k} \neq 0\right\}$, then $k_{0}=0$ as otherwise we have terms with $e^{-\left(k_{0}+1\right) S_{1}}$ that can't be eliminated by any other term.

The proof for all other cases $e \mathcal{H}_{I V} e, e \mathcal{H}_{I I I} e, e \mathcal{H}_{I I} e$ follows the same reasoning and we omit it. 
To prove the statement in the case of the two algebras $e \mathcal{H}_{I I I^{D_{7}}} e$ and $e \mathcal{H}_{I I I^{D_{8}}} e$, we observe that they are special cases of $e \mathcal{H}_{I I I} e$ for $b=0$ and $a=b=0$ respectively. Bearing this in mind, we can prove that the two algebras $e \mathcal{H}_{I I I^{D_{7}}} e$ and $e \mathcal{H}_{I I I^{D_{8}}} e$ can be embedded in to $\operatorname{Mat}_{2}\left(\mathbb{T}_{q}\right)$ and follow the same reasoning as above.

Now in each case we give a faithful representation the confluent Zhedanov algebras either on the space of symmetric Laurent polynomials $\mathcal{L}_{\text {sym }}$ or on the space of polynomials $\mathcal{P}$. In order to prove that our representation is faithful, we need first the following lemma:

Lemma 6.3. The quotiented Zhedanov algebra $\mathcal{Z}_{d} /\left\langle\mathcal{C}=\mathcal{C}_{0}\right\rangle$, has elements

$$
K_{0}^{n}\left(K_{1} K_{0}\right)^{l} K_{1}^{m}, \quad m, n=0,1,2,3, \ldots, \quad l=0,1
$$

as a basis.

Proof. The proof is the same as in the non-confluent case see the results contained in Section 2 of [24].

We will give the proofs only in the first case, i.e. for $\mathcal{Z}_{V}$. All other proofs are similar and therefore will be omitted for brevity.

\subsection{Representation of $\mathcal{Z}_{V}$ and continuous dual q-Hahn polynomials.}

Lemma 6.4. The confluent Zhedanov algebra $\mathcal{Z}_{V} /\left\langle\mathcal{C}=\mathcal{C}_{0}\right\rangle$ admits the following representation on the space $\mathcal{L}_{\text {sym }}$ of symmetric Laurent polynomials:

$$
\begin{aligned}
\left(K_{1} f\right)[x]:= & \left(x+\frac{1}{x}\right) f[x], \\
\left(K_{0} f\right)[x]:= & \frac{(1-a x)(1-b x)(1-c x)}{\left(1-x^{2}\right)\left(1-q x^{2}\right)}(f[q x]-f[x])+f[x]- \\
& -x \frac{(a-x)(b-x)(c-x)}{\left(1-x^{2}\right)\left(q-x^{2}\right)}\left(f\left[q^{-1} x\right]-f[x]\right) .
\end{aligned}
$$

Proof. By expressing the confluent Zhedanov algebra structure constants by the parameters $a, b, c$ : it is a straightforward computation (see notebook 8 in [28]) to prove that the operators satisfy the relations $(1.49,1.50,1.51)$, with constants $B, C_{1}, D_{0}, D_{1}$ given in table 1.

Lemma 6.5. The continuous dual q-Hahn polynomials:

$$
p_{n}(x ; a, b, c, d):=\frac{(a b, a c ; q)_{n}}{a^{n}}{ }_{3} \phi_{2}\left(\begin{array}{c}
q^{-n}, a x, a x^{-1} \\
a b, a c,
\end{array} ; q, q\right),
$$

where $a=-\frac{u_{1}}{k_{1}}, b=u_{1} k_{1}, c=-\frac{\sqrt{q}}{u_{0}}$, are eigenfunctions of the $K_{0}$ operator:

$$
K_{0} p_{n}=q^{-n} p_{n}
$$

Proof. Note that the confluent Zhedanov algebra $\mathcal{Z}_{V} /\left\langle\mathcal{C}=\mathcal{C}_{0}\right\rangle$ is obtained as the limit for $d \rightarrow 0$ of the general Zhedanov algebra $\mathcal{Z} /\left\langle\mathcal{C}=\mathcal{C}_{0}\right\rangle$. Analogously, the representation $(6.216,6.217)$ is the limit as $d \rightarrow 0$ of the representation $(2.58,2.59)$ of the general Zhedanov algebra and the continuous dual $q$-Hahn polynomials are obtained as limit for $d \rightarrow 0$ of the Askey-Wilson polynomilas. As a consequence this proof follows from the fact that the Askey-Wilson polynomials are eigenfunctions of the operator $K_{0}$ defined by $(2.59)$.

Lemma 6.6. The representation (6.216,6.217) is faithful. 
Proof. This proof follows the same lines as the proof of Theorem 2.2 in [24], where we replace the Askey-Wilson polynomials by the dual $q$-Hahn polynomials.

6.2. Big $q-$ Jacobi polynomials. In order to understand the relation between the confluent Zhedanov algebra $\mathcal{Z}_{V}$ and the Big q-Jacobi polynomials, we use the confluent version of the Cherednik algebra automorphism $\gamma$ defined in sub-section 2.1. As shown in Section 3.1, in the confluent limit, $\gamma$ defines an algebra isomorphism (3.109) mapping $\mathcal{H}_{V}$ to $\mathcal{H}_{V}^{\gamma}$ given by $(3.110,3.1113 .1123 .1133 .114)$. On the confluent Zhedanov algebra we obtain the following result:

Lemma 6.7. The transformation

$$
\gamma\left(K_{0}, K_{1}\right)=\left(k_{1} u_{1} K_{1}, \frac{1}{u_{1}}\left(K_{0}+\frac{q^{\frac{3}{2}}}{(q+1)(q-1)^{2}}\left[K_{2}, K_{1}\right]\right)\right),
$$

is an isomorphism mapping $\mathcal{Z}_{V}$ to $\mathcal{Z}_{V}^{\gamma}$, which is the algebra generated by $K_{0}^{\gamma}, K_{1}^{\gamma}$ with relations:

(6.218) $\left(q+q^{-1}\right) K_{1}^{\gamma} K_{0}^{\gamma} K_{1}^{\gamma}-\left(K_{1}^{\gamma}\right)^{2} K_{0}^{\gamma}-K_{0}^{\gamma}\left(K_{1}^{\gamma}\right)^{2}=B^{\gamma} K_{1}^{\gamma}+D_{0}^{\gamma}$,

$$
\left(q+q^{-1}\right) K_{0}^{\gamma} K_{1}^{\gamma} K_{0}^{\gamma}-\left(K_{0}^{\gamma}\right)^{2} K_{1}^{\gamma}-K_{1}^{\gamma}\left(K_{0}^{\gamma}\right)^{2}=B^{\gamma} K_{0}^{\gamma}+C_{1}^{\gamma} K_{1}^{\gamma}+D_{1}^{\gamma} \text {, }
$$

for some arbitrary constants $B^{\gamma}, C_{1}^{\gamma}, D_{0}^{\gamma}$ and $D_{1}^{\gamma 6}$.

Proof. It is a straightforward computation to prove that on the spherical subalgebra $e \mathcal{H}_{V} e$ the isomorphism $\gamma$ acts as follows:

$$
\gamma\left(\hat{X}_{1}, \hat{X}_{2}, \hat{X}_{3}\right)=\left(\frac{\sqrt{q}}{q-1}\left[\hat{X}_{3}, \hat{X}_{1}\right]+\hat{X}_{2}, \hat{X}_{1}, \hat{X}_{3}\right) .
$$

By using the isomorphism $i: \mathcal{Z}_{V} /\left\langle\mathcal{C}=\mathcal{C}_{0}\right\rangle \rightarrow e \mathcal{H}_{V} e$ obtained in theorem 6.2 we can deduce the result.

Lemma 6.8. The confluent Zhedanov algebra $\mathcal{Z}_{V}^{\gamma} /\left\langle\mathcal{C}=\mathcal{C}_{0}\right\rangle$ admits the following representation on the space $\mathcal{P}$ of polynomials:

$$
\begin{aligned}
\left(K_{1}^{\gamma} f\right)[x]:= & x f[x], \\
\left(K_{0}^{\gamma} f\right)[x]:= & \frac{q(\lambda \tilde{c} x+\tilde{a}(x(1+\tilde{b})-\tilde{c}(1+q-\lambda x)))}{\lambda^{2} x^{2}} f[x]+ \\
& +\frac{(\lambda x-q \tilde{a})(\lambda x-q \tilde{c})}{\lambda^{2} x^{2}} f\left[\frac{x}{q}\right]+\frac{q(\lambda x-1) \tilde{a}(\tilde{b} \lambda x-\tilde{c})}{x^{2}} f[q x] .
\end{aligned}
$$

Proof. Indeed the generators defined by $(6.219,6.220)$ satisfy the relations $(6.218)$ for

$$
\begin{gathered}
B^{\gamma}=(q-1)^{2} \frac{\tilde{c}+\tilde{a}(1+\tilde{b}+\tilde{c})}{\lambda}, \quad D_{0}^{\gamma}=-(q+1)(q-1)^{2} \frac{\tilde{a} \tilde{c}}{\lambda^{2}} \\
C_{1}^{\gamma}=q\left(q-\frac{1}{q}\right)^{2} \tilde{a} \tilde{b}, \quad D_{1}^{\gamma}=-(q-1)^{2}(q+1) \frac{\tilde{a}(\tilde{c}+\tilde{b}(1+\tilde{a}+\tilde{c}))}{\lambda} .
\end{gathered}
$$

see notebook 9 in [28]).

The proof of the following two results is obtained by taking substituting $x \rightarrow \frac{x}{\varepsilon}$, $a \rightarrow \varepsilon \lambda, b \rightarrow \frac{\tilde{a} q}{\varepsilon \lambda}, c \rightarrow \frac{\tilde{c} q}{\varepsilon \lambda}, d \rightarrow \varepsilon \lambda \frac{\tilde{b}}{\tilde{c}}$ and taking the limit as $\varepsilon \rightarrow 0$ in the analogous results for the Askey Wilson polynomials in [24].

\footnotetext{
${ }^{6}$ Only three of these are independent, as one constant can be rescaled to any arbitrary value by rescaling the generators.
} 
Lemma 6.9. The big q-Jacobi polynomials:

$$
P_{n}(x ; a, b, c, d):={ }_{3} \phi_{2}\left(\begin{array}{c}
q^{-n}, a b q^{n+1}, x \\
a q, c q,
\end{array} ; q, q\right),
$$

are eigenfunctions of the $K_{0}$ operator:

$$
K_{0} P_{n}[\lambda x]=\frac{1+q^{2 n+1} a b}{q^{n}} P_{n}[\lambda x] .
$$

Lemma 6.10. The representation (6.219,6.220) is faithful.

Remark 6.11. We have introduced an arbitrary parameter $\lambda$ in our representation to account for the freedom of direction of confluence. This freedom will be used later on to produce exact matches between parameters in the polynomials and parameters in the confluent Zhedanov algebras,

6.3. Representation of $\mathcal{Z}_{I V}$ and Big $q$-Laguerre Polynomials. Note that the algebra $\mathcal{H}_{I V}$ can be obtained as limit of the algebra $\mathcal{H}_{V}^{\gamma}$ by rescaling $V_{0}^{\gamma} \rightarrow \frac{1}{\varepsilon} V_{0}^{\gamma}$, $\check{V}_{0}^{\gamma} \rightarrow \frac{1}{\varepsilon} \check{V}_{0}^{\gamma}, k_{0} \rightarrow \varepsilon, u_{0} \rightarrow \varepsilon u_{0}$. This shows that the confluent Zhedanov algebra $\mathcal{Z}_{I V}$ can be obtained from $\mathcal{Z}_{V}^{\gamma}$ in the limit $b \rightarrow 0$. This leads to the following results:

Lemma 6.12. The confluent Zhedanov algebra $\mathcal{Z}_{I V} /\left\langle\mathcal{C}=\mathcal{C}_{0}\right\rangle$ admits the following representation on the space $\mathcal{P}$ of polynomials:

$$
\begin{aligned}
\left(K_{1} f\right)[x]:= & x f[x], \\
\left(K_{0} f\right)[x]:= & \frac{q(\lambda \tilde{c} x+\tilde{a}(x-\tilde{c}(1+q-\lambda x)))}{\lambda^{2} x^{2}} f[x]+ \\
& +\frac{(\lambda x-q \tilde{a})(\lambda x-q \tilde{c})}{\lambda^{2} x^{2}} f\left[\frac{x}{q}\right]-\frac{q(\lambda x-1) \tilde{a} \tilde{c}}{x^{2}} f[q x] .
\end{aligned}
$$

Proof. Indeed the generators defined by $(6.221,6.222)$ satisfy the relations $(6.214)$ for

$$
\begin{gathered}
B=(q-1)^{2} \frac{\tilde{c}+\tilde{a}(1+\tilde{c})}{\lambda}, \quad D_{0}^{\gamma}=-(q+1)(q-1)^{2} \frac{\tilde{a} \tilde{c}}{\lambda^{2}}, \\
D_{1}=-(q-1)^{2}(q+1) \frac{\tilde{a} \tilde{c}}{\lambda},
\end{gathered}
$$

(see notebook 10 in [28]). Choosing $\tilde{a}=-\frac{b^{2}}{q}, \tilde{c}=-\frac{b c}{q}, \lambda=-b$, we find that these formulae are the same as the ones in Table 1.

Lemma 6.13. The big q-Laguerre polynomials:

$$
P_{n}(x ; \tilde{a}, \tilde{c}, q):={ }_{3} \phi_{2}\left(\begin{array}{c}
q^{-n}, 0, x \\
\tilde{a} q, \tilde{c} q,
\end{array} ; q, q\right),
$$

are eigenfunctions of the $K_{0}$ operator:

$$
K_{0} P_{n}[\lambda x]=q^{-n} P_{n}[\lambda x] .
$$

Lemma 6.14. The representation (6.221,6.222) is faithful. 


\subsection{Representation of $\mathcal{Z}_{I I I}$ and Al-Salam-Chihara Polynomials.}

Lemma 6.15. The confluent Zhedanov algebra $\mathcal{Z}_{I I I}$ admits the following representation on the space $\mathcal{L}_{\text {sym }}$ of symmetric Laurent polynomials:

$$
\begin{aligned}
\left(K_{1} f\right)[x]:= & \left(x+\frac{1}{x}\right) f[x], \\
\left(K_{0} f\right)[x]:= & x \frac{(1-a x)(1-b x)}{\left(1-x^{2}\right)\left(1-q x^{2}\right)}(f[q x]-f[x])+ \\
& +x \frac{(a-x)(b-x)}{\left(1-x^{2}\right)\left(q-x^{2}\right)}\left(f\left[q^{-1} x\right]-f[x]\right) .
\end{aligned}
$$

Proof. By expressing the confluent Zhedanov algebra structure constants by the parameters $a, b, c$ as in Table 1 , it is a straightforward computation (see notebook 11 in [28]) to prove that the operators $(6.223,6.224)$ satisfy $(6.214)$.

Lemma 6.16. The Al-Salam-Chihara polynomials:

$$
Q_{n}(x ; a, b, d):=\frac{(a b ; q)_{n}}{a^{n}}{ }_{3} \phi_{2}\left(\begin{array}{c}
q^{-n}, a x, a x^{-1} \\
a b, 0,
\end{array} ; q, q\right),
$$

are eigenfunctions of the following operator

$$
K_{0}^{\beta}:=\frac{q}{q^{2}-1}\left(K_{0} K_{1}-q K_{1} K_{0}-\frac{(a+b)(q-1)}{q}\right),
$$

with eigenvalues

$$
\frac{1}{q^{n}}-1+\frac{1+a+b-a b}{q+1}
$$

Remark 6.17. Note that now the operator $K_{0}$ does not act nicely on the Al-SalamChihara polynomials; we had to replace it by the new operator $K_{0}^{\beta}$. This is due to the fact that in terms of generators $T_{0}, T_{1}, X, W$ and parameters $a, b$, the algebra $\mathcal{H}_{I I I}$ is obtained as a limiting case of $\mathcal{H}_{V}$ for $c \rightarrow \infty$, while the Al-Salam-Chihara polynomials are obtained by the continuous dual $q$-Hahn polynomials in the limit $c \rightarrow 0$. In order to match these two asymptotics, we need to perform the following transformation of the $\mathcal{H}_{I I I}$ algebra:

$$
\beta\left(T_{0}, T_{1}, X\right)=\left(\sqrt{q} W T_{0}-1, T_{1}, X\right) .
$$

This transformation is an isomorphism between the algebra $\mathcal{H}_{I I I}$ in the representation $(1.16, \ldots, 1.20)$ and the algebra $\mathcal{H}_{I I I}^{\beta}$ generated by $T_{0}, T_{1}, X, W$ and relations (1.16), (1.17), (1.19) and the algebra:

$$
\begin{array}{r}
T_{0}^{2}+T_{0}=0, \\
q T_{0} W=X\left(T_{0}+1\right),
\end{array}
$$

which is obtained from the $\mathcal{H}_{V}$ algebra as $c \rightarrow 0$. This transformation acts on the confluent Zhedanov algebra $\mathcal{Z}_{I I I}$ as follows:

$$
\beta\left(K_{0}, K_{1}\right)=\left(K_{0}^{\beta}, K_{1}\right) .
$$

Lemma 6.18. The representation (6.223,6.224) is faithful.

Proof. We use the same idea as in the remark above and prove all statements as limits for $c \rightarrow 0$ of the analogous statements for the algebra $\mathcal{H}_{V}$ and the dual q-Hahn polynomials. 
6.5. Representation of $\mathcal{Z}_{I I I}^{D_{7}}$ and continuous Big $q$-Hermite Polynomials. Note that the algebra $\mathcal{H}_{I I I}^{D_{7}}$ can be obtained as limit of the algebra $\mathcal{H}_{I I I}$ by taking the limit $b \rightarrow 0$ and $c \rightarrow 1$ (see notebook 12 in [28]). This leads to the following results:

Lemma 6.19. The confluent Zhedanov algebra admits the following representation on the space $\mathcal{L}_{\text {sym }}$ of symmetric Laurent polynomials:

$$
\begin{aligned}
\left(K_{1} f\right)[x]:= & \left(x+\frac{1}{x}\right) f[x], \\
\left(K_{0} f\right)[x]:= & x \frac{(1-a x)}{\left(1-x^{2}\right)\left(1-q x^{2}\right)}(f[q x]-f[x])- \\
& x^{2} \frac{(a-x)}{\left(1-x^{2}\right)\left(q-x^{2}\right)}\left(f\left[q^{-1} x\right]-f[x]\right) .
\end{aligned}
$$

Lemma 6.20. The continuous big q-Hermite polynomials:

$$
H_{n}(x ; a, b, c, d):=\frac{1}{a^{n}}{ }^{3} \phi_{2}\left(\begin{array}{c}
q^{-n}, a x, a x^{-1} \\
0,0,
\end{array} ; q, q\right),
$$

are eigenfunctions of the following operator

$$
K_{0}^{\beta}:=\frac{q}{q^{2}-1}\left(K_{0} K_{1}-q K_{1} K_{0}-\frac{a(q-1)}{q}\right),
$$

with eigenvalues

$$
\frac{1}{q^{n+1}}-\frac{1+a}{q+1}
$$

Lemma 6.21. The representation (6.227,6.228) is faithful.

6.6. Representation of $\mathcal{Z}_{I I}^{D_{8}}$ and continuous $q$-Hermite Polynomials. Note that the algebra $\mathcal{H}_{I I I}^{D_{8}}$ can be obtained as limit of the algebra $\mathcal{H}_{I I I}^{D_{7}}$ by taking the limit $a \rightarrow 0$. This leads to the following results (see notebook 13 in [28]):

Lemma 6.22. The confluent Zhedanov algebra $\mathcal{Z}_{I I I}^{D_{8}}$ admits the following representation on the space $\mathcal{L}_{\text {sym }}$ of symmetric Laurent polynomials:

$$
\begin{aligned}
\left(K_{1} f\right)[x]:= & \left(x+\frac{1}{x}\right) f[x], \\
\left(K_{0} f\right)[x]:= & x \frac{1}{\left(1-x^{2}\right)\left(1-q x^{2}\right)}(f[q x]-f[x])+ \\
& x^{3} \frac{1}{\left(1-x^{2}\right)\left(q-x^{2}\right)}\left(f\left[q^{-1} x\right]-f[x]\right) .
\end{aligned}
$$

Lemma 6.23. The continuous $q$-Hermite polynomials:

$$
H_{n}(x ; a, b, c, d):=x_{2}^{n} \phi_{0}\left(\begin{array}{c}
q^{-n}, 0 \\
-
\end{array} ;, \frac{q^{n}}{x^{2}}\right),
$$

are eigenfunctions of the following operator

$$
K_{0}^{\beta}:=\frac{q}{q^{2}-1}\left(K_{0} K_{1}-q K_{1} K_{0}\right),
$$

with eigenvalues

$$
\frac{1}{q^{n+1}}-\frac{1}{q+1}
$$

Lemma 6.24. The representation (6.229,6.230) is faithful. 


\subsection{Representation of $\mathcal{Z}_{I I}$ and little $q$-Laguerre/Wall polynomials.}

Lemma 6.25. The confluent Zhedanov algebra $\mathcal{Z}_{I I} /\left\langle\mathcal{C}=\mathcal{C}_{0}\right\rangle$ admits the following representation on the space $\mathcal{P}$ of polynomials:

$$
\begin{aligned}
& \left(K_{1} f\right)[x]:=x f[x], \\
& \left(K_{0} f\right)[x]:=\frac{1+\tilde{a}}{\lambda x} f[x]+\frac{\lambda x-1}{\lambda x} f\left[\frac{x}{q}\right]-\frac{\tilde{a}}{\lambda x} f[q x] .
\end{aligned}
$$

Proof. Indeed the generators defined by $(6.231,6.232)$ satisfy the relations $(6.214)$ with

$$
B=\frac{(q-1)^{2}(1+\tilde{a})}{\lambda q}, \quad D_{1}=-\frac{(q-1)^{2}(1+q)}{\lambda q} \tilde{a} .
$$

Choosing $\tilde{a}=-\frac{b^{2}}{q}, \lambda=-b$, we find that these formulae are the same as the ones in Table 1 .

The following results can be proved by taking $c \rightarrow-\frac{1}{\varepsilon}$ and $x \rightarrow \frac{q x}{\varepsilon}$ and letting $\varepsilon \rightarrow 0$ in the results proved for $\mathcal{Z}_{I V}$ (see also notebook 14 in [28]).

Lemma 6.26. The little q-Laguerre polynomials:

$$
p_{n}(x ; a, c, d):={ }_{3} \phi_{2}\left(\begin{array}{c}
q^{-n}, 0 \\
a q,
\end{array} ; q, q x\right),
$$

are eigenfunctions of the $K_{0}$ operator:

$$
K_{0} p_{n}[x]=q^{-n} p_{n}[x] .
$$

Lemma 6.27. The representation (6.231,6.232) is faithful.

6.8. Representation of $\mathcal{Z}_{I}$ and a special case of the little $q$-Laguerre/Wall polynomials. The confluent Zhedanov algebra $\mathcal{Z}_{I}$ can be obtained from $\mathcal{Z}_{I I}$ in the limit $a \rightarrow 0$. This leads to the following results:

Lemma 6.28. The confluent Zhedanov algebra $\mathcal{Z}_{I} /\left\langle\mathcal{C}=\mathcal{C}_{0}\right\rangle$ admits the following representation on the space $\mathcal{P}$ of polynomials:

$$
\begin{aligned}
& \left(K_{1} f\right)[x]:=x f[x], \\
& \left(K_{0} f\right)[x]:=\frac{1}{x} f[x]+\frac{x-1}{x} f\left[\frac{x}{q}\right],
\end{aligned}
$$

with

$$
B=\frac{(q-1)^{2}}{q}
$$

Lemma 6.29. The little $q$-Laguerre polynomials with $a=0$ :

$$
p_{n}(x ; 0, c, d):={ }_{3} \phi_{2}\left(\begin{array}{c}
q^{-n}, 0 \\
0,
\end{array} ; q, q x\right),
$$

are eigenfunctions of the $K_{0}$ operator:

$$
K_{0} p_{n}[x]=q^{-n} p_{n}[x] .
$$

Lemma 6.30. The representation (6.233,6.234) is faithful. 


\section{Appendix A The Painlevé monodromy manifolds}

All the Painlevé differential equations arise as monodromy preserving deformations of an auxiliary system of two first order ODEs [21, 22, 12, 13]. The monodromy data of such auxiliary system are encoded in their monodromy manifolds which can all be described by cubic surfaces in $\mathbb{C}^{3}$ defined by the zero locus of the following polynomials in $\mathbb{C}\left[x_{1}, x_{2}, x_{3}\right]$ :

$$
\begin{aligned}
& \text { PVI } \quad x_{1} x_{2} x_{3}+x_{1}^{2}+x_{2}^{2}+x_{3}^{2}-\left(G_{1} G_{\infty}+G_{2} G_{3}\right) x_{1}-\left(G_{2} G_{\infty}+G_{1} G_{3}\right) x_{2}- \\
& P V \quad x_{1} x_{2} x_{3}+x_{1}^{2}+x_{2}^{2}-\left(G_{1} G_{\infty}+G_{2} G_{3}\right) x_{1}-\left(G_{2} G_{\infty}+G_{1} G_{3}\right) x_{2}- \\
& -G_{3} G_{\infty} x_{3}+G_{\infty}^{2}+G_{3}^{2}+G_{1} G_{2} G_{3} G_{\infty} \\
& \text { PIV } \quad x_{1} x_{2} x_{3}+x_{1}^{2}-\left(G_{1} G_{\infty}+G_{2} G_{3}\right) x_{1}-G_{2} G_{\infty} x_{2}-G_{3} G_{\infty} x_{3}+ \\
& +G_{\infty}^{2}+G_{1} G_{2} G_{3} G_{\infty} \\
& \text { PIII } \quad x_{1} x_{2} x_{3}+x_{1}^{2}+x_{2}^{2}-G_{1} G_{\infty} x_{1}-G_{2} G_{\infty} x_{2}+G_{\infty}^{2}, \\
& P I I I^{D_{7}} \quad x_{1} x_{2} x_{3}+x_{1}^{2}+x_{2}^{2}-G_{1} G_{\infty} x_{1}-G_{2} G_{\infty} x_{2}, \\
& P I I I^{D_{8}} \quad x_{1} x_{2} x_{3}+x_{1}^{2}+x_{2}^{2}-G_{2} G_{\infty} x_{2}, \\
& P I I \quad x_{1} x_{2} x_{3}+x_{1}^{2}-G_{1} G_{\infty} x_{1}-G_{2} G_{\infty} x_{2}+G_{\infty}^{2} \\
& \text { PI } \quad x_{1} x_{2} x_{3}-G_{1} G_{\infty} x_{1}-G_{2} G_{\infty} x_{2}+G_{\infty}^{2},
\end{aligned}
$$

where $G_{1}, G_{2}, G_{3}, G_{\infty}$ are some constants related to the parameters appearing in the corresponding Painlevé equation as described in [6]. Note that not all parameters are independent is the above cubic polynomials: $G_{\infty}$ can be chosen arbitrarily in the $P V, P I I I, P I I I^{D_{7}}, P I I I^{D_{8}}, P I I$ and $P I$ cubics, $G_{2}$ can be chosen arbitrarily in the $P I I I^{D_{7}}, P I V, P I I, P I$ cubics, $G_{3}$ can be chosen arbitrarily in the $P I I$, and $P I$ cubics. To match these cubics to the ones found as semiclassical limits of the spherical sub-algebras of the confluent Cherednik algebras defined in this paper we need to perform the following substitutions:

$$
\begin{aligned}
& x_{1}=\left\{\begin{array}{cc}
-X_{2} & \text { for } P V I, P V, P I I I, P I I I^{D_{7}}, P I I I^{D_{8}}, \\
-X_{3} & \text { for } P I V, P I I, P I,
\end{array}\right. \\
& x_{2}=\left\{\begin{array}{cc}
-X_{3} & \text { for } P V I, P V, P I I I, P I I I^{D_{7}}, P I I I^{D_{8}}, \\
-X_{1} & \text { for } P I V, P I I, P I,
\end{array}\right. \\
& x_{3}=\left\{\begin{array}{lc}
-X_{1} & \text { for } P V I, P V, P I I I, P I I I^{D_{7}}, P I I I^{D_{8}}, \\
-X_{2} & \text { for } P I V, P I I, P I,
\end{array}\right. \\
& G_{\infty}=\left\{\begin{array}{lc}
-i \bar{k}_{0} & \text { for } P V I, \\
i & \text { for } P V, P I I I, P I I I^{D_{7}}, P I I I^{D_{8}}, P I I \\
i c & \text { for } P I V, \\
1 & \text { for } P I,
\end{array}\right.
\end{aligned}
$$




$$
\begin{gathered}
G_{3}=\left\{\begin{array}{lc}
-i \bar{u}_{0} & \text { for } P V I \\
-i c & \text { for } P V \\
0 & \text { for } P I I I, P I I^{D_{7}}, P I I I^{D_{8}}, P I I, P I \\
-i & \text { for } P I V,
\end{array}\right. \\
G_{2}=\left\{\begin{array}{lc}
-i \bar{k}_{1} & \text { for } P V I, P V, \\
i\left(\sqrt{a b}+\frac{1}{\sqrt{a b}}\right) & \text { for } P I I I, \\
i & \text { for } P I I^{D_{7}}, P I I I^{D_{8}}, P I I, \\
-i & \text { for } P I V, \\
-1 & \text { for } P I, \\
-i \bar{u}_{1} & \text { for } P V I, P V, \\
i\left(\sqrt{\frac{b}{a}}+\sqrt{\frac{a}{b}}\right) & \text { for } P I I I, \\
-i\left(b-\frac{1}{b}\right) & \text { for } P I V, \\
i\left(b-\frac{1}{b}\right) & \text { for } P I I, \\
-i a & \text { for } P I I I^{D_{7}} \\
0 & \text { for } P I I I^{D_{8}} \\
1 & \text { for } P I .
\end{array}\right.
\end{gathered}
$$

Acknowledgements. The author is specially grateful to T. Koornwinder for reading trough this paper and coming up with many useful comments. The author wishes to thank also M. Balagovic, Yu. Berest, O. Chalyck, F. Eshmatov, P. Etingof, N. Joshi, C. Korff, M. Noumi, V. Rubtsov, J. Stokman and P. Terwilliger for interesting discussions on this subject. This research was supported by the EP$\mathrm{SRC}$ and by the Hausdorff Institute. The author wishes to thank the referees for their useful comments.

\section{REFERENCES}

[1] Askey R., Wilson J. A., Some basic hypergeometric orthogonal polynomials that generalise Jacobi polynomials, Memoirs of the AMS, 319 (1985).

[2] Berest Y., Etingof P., Ginzburg V., Finite-dimensional representations of rational Cherednik algebras. Int. Math. Res. Not. 2003 no. 19:1053-1088.

[3] Bleher P. and Its A., Asymptotics of orthogonal polynomials, Riemann-Hilbert problem, and universality in the matrix model, Ann. of Math. (2) 150 (1999), no. 1:185-266.

[4] Borodin A. and Deift P., Fredholm determinants, Jimbo-Miwa-Ueno $\tau$-functions, and representation theory, Commun. Pure Appl. Math. 55 (2002) 1160-1230.

[5] Chekhov L., Mazzocco M., Shear coordinate description of the quantised versal unfolding of a $D_{4}$ singularity, J. Phys. A: Math. Theor. 43, (2010) 442002, 13 pages.

[6] Chekhov L., Mazzocco M., Rubtsov V., Painlevé monodromy manifolds, decorated character varieties and cluster algebras, In progress, (2015)

[7] Cherednik I., Double affine Hecke algebras, Knizhnik-Zamolodchikov equations and Macdonald's operators, Int. Math. Res. Not. (1992), no.9:171-180.

[8] Cherednik I., Whittaker limits of difference spherical functions, Int. Math. Res. Not. (2009), no.20:3793-3842.

[9] Clarkson P. A., Special polynomials associated with rational solutions of the Painlevé equations and applications to soliton equations, Comput. Methods Funct. Theory 6 (2006), no. 2:329-401.

[10] Eshmatov A. and Eshmatov F., Notes on isomorphism between skein algebra and DAHA, private communication, (2012).

[11] Etingof P., Oblomkov A. and Rains E., Generalised double affine Hecke algebras of rank 1 and quantised del Pezzo surfaces, Adv. Math., 212 (2007), 749-796. 
[12] Fokas A. S., Its A. R., Kapaev A., Novokshenov V. Yu., Painlevé transcendents. The RiemannHilbert approach, Mathematical Surveys and Monographs, 128 American Mathematical Society, Providence, RI (2006). xii+553 pp. ISBN: 0-8218-3651-X.

[13] Fokas A. S., Its A. R., The isomonodromy method and the Painlevé equations, Important developments in soliton theory, Springer Ser. Nonlinear Dynam., Springer, Berlin, (1993) 99-122.

[14] Forrester, P. J.; Witte, N. S., Application of the $\tau$-function theory of Painlevé equations to random matrices: PIV, PII and the GUE, Comm. Math. Phys. 219 (2001), no. 2:357-398.

[15] Fuchs R., Ueber lineare homogene Differentialgleichungen zweiter Ordnung mit drei im Endlichen gelegenen wesentlich singulären, Stellen. Math. Ann., 63 (1907) 301-321.

[16] Garnier R., Solution du probleme de Riemann pour les systemes différentielles linéaires du second ordre, Ann. Sci. Ecole Norm.. Sup., 43 (1926) 239-352.

[17] Gasper G., Raman M., Basic hypergeometric series, Encyclopedia of Mathematics and Its Applications, 35, Cambridge University Press (1990).

[18] Gordon I., Baby Verma modules for rational Cherednik algebras, Bull. London Math. Soc.35 (2003), no. 3:321-336.

[19] Ito T. and Terwillinger P., Double affine Hecke algebras of rank 1 and the $\mathbb{Z}_{3}$-symmetric Askey-Wilson relations, SIGMA 6 (2010) 065, 9 pages.

[20] Jimbo M., Monodromy Problem and the Boundary Condition for Some Painlevé Equations, Publ. RIMS, Kyoto Univ., 18 (1982) 1137-1161.

[21] Jimbo M., Miwa T. and Ueno K., Monodromy preserving deformations of linear ordinary differential equations with rational coefficients I, Physica 2D, 2, (1981), no. 2, 306-352

[22] Jimbo M. and Miwa T., Monodromy preserving deformations of linear ordinary differential equations with rational coefficients II, Physica 2D, 2 (1981), no. 3, 407-448.

[23] Koekoek R., Lesky P., Swarttouw R. F., Hypergeometric Orthogonal Polynomials and Their $q$-Analogues, Springer Monographs in Mathematics, Springer-Verlag, Berlin, (2010).

[24] Koornwinder T. H., The relationship between Zhedanov's algebra $\mathcal{Z}$ and the double affine Hecke algebra in the rank one case, SIGMA 3 (2007), 063, 15 pp.

[25] Koornwinder T. H., Zhedanov's algebra $\mathcal{Z}$ and the double affine Hecke algebra in the rank one case. II. The spherical subalgebra, SIGMA 4 (2008), 052, 17 pp.

[26] Koornwinder T. H. and Bouzeffour F., Nonsymmetric Askey-Wilson polynomials as vectorvalued polynomials, Appl. Anal., 90 (2011), 731-746.

[27] Koornwinder T. H., Duality between Big q-Jacobi and continuous dual q-Hahn polynomials, private communication, (2013).

[28] Mazzocco M., Mathematica notebooks, on http://homepages.lboro.ac.uk/ mamm4/notebooks.html.

[29] Mazzocco M., Non-Symmetric Basic Hypergeometric Polynomials and Representation Theory for Confluent Cherednik Algebras, SIGMA 10 (2014), 116, 10 pp.

[30] Mazzocco M., Embedding of the rank 1 DAHA into $\operatorname{Mat}\left(2, \mathbb{T}_{q}\right)$ and its automorphisms. arXiv:1603.03770 To appear in Advanced Studies in Pure Mathematics.

[31] Hurst D., Lamm D., Merino O., Obar R., Pfister H., Walker M., Wavrik J., Yu L., Camino J., Griffin J., Ovall J., Shaheen T., Shopple J., NCAlgebra, a non-commutative algebra package running under Mathematica, http://www.math.ucsd.edu/ ncalg/.

[32] M. Noumi, S. Okada, K. Okamoto and H. Umemura, Special polynomials associated with the Painlevé equations. II, in: Integrable Systems and Algebraic Geometry, M.-H. Saito, Y. Shimizu and R. Ueno (eds.), World Scientific, Singapore (1998) 349-372.

[33] Noumi M., and Stokman J. V., Askey-Wilson polynomials: an affine Hecke algebraic approach, Laredo Lectures on Orthogonal Polynomials and Special Functions, Adv. Theory Spec. Funct. Orthogonal Polynomials, Nova Sci. Publ., Hauppauge, NY (2004) 111-144.

[34] Noumi M., Yamada Y., Symmetries in the fourth Painlevé equation and Okamoto polynomials, Nagoya Math. J. 153 (1999), 53-86.

[35] Oblomkov A., Double affine Hecke algebras of rank 1 and affine cubic surfaces, IMRN 2004, no. 18:877-912.

[36] Okamoto K., On the $\tau$-function of the Painlevé equations, Physica D2 (1981), 525-535.

[37] Okamoto K., Studies on the Painlevé equations III. Second and fourth Painlevé equations, PII and PIV, Math. Ann. 275 (1986), 221-255.

[38] Okamoto K., Studies on the Painlevé equations IV. Third Painlevé equation PIII, Funkcial. Ekvac. 30 (1987), 305-332. 
[39] Sahi S. Nonsymmetric Koornwinder polynomials and duality, Selecta Math. (N.S.) 150, (1999) no1:267-282.

[40] Saito M., van der Put M., Moduli spaces for linear differential equations and the Painlevé equations, Ann. Inst. Fourier (Grenoble) 59 (2009), no. 7:2611-2667.

[41] Sakai H.,Rational Surfaces Associated with Affine Root Systems and Geometry of the Painlevé Equations, Commun. Math. Phys. 220 (2001) 165-229.

[42] Schlesinger L., Über eine Klasse von Differentialsystemen beliebiger Ordnung mit festen kritischen Punkten, J. Reine Angew. Math., 141, (1912), 96-145.

[43] Stokman J. V., Difference Fourier transforms for nonreduced root systems, Selecta Math. (N.S.) 9 (2003) no3:409-494.

[44] Terwilliger, P. The universal Askey-Wilson algebra, SIGMA 7 (2011), 069, 24 pages, arXiv:1104.2813.

[45] Terwilliger, P. The Universal Askey-Wilson Algebra and DAHA of Type $\left(C_{1}^{\vee}, C_{1}\right), S I G M A$ 9 (2013), 047, 40 pages. arXiv: 1202.4673

[46] Tracy,C.A. and Widom,H., Fredholm Determinants, Differential Equations and Matrix Models, Commun. Math. Phys. 163 (1994) 33-72.

[47] Umemura H., Painlevé equations in the past 100 Years, A.M.S. Translations 204 (2001), $81-110$.

[48] van der Put M., Families of Linear Differential Equations and the Painlevé equations, Séminaires et Congrés, 27 (2013) 203-220.

[49] Vorobev A. P., On rational solutions of the second Painlevé equation, Diff. Eqns. 1 (1965), $58-59$.

[50] Yablonskii A. I., On rational solutions of the second Painlevé equation, Vesti Akad. Navuk. BSSR Ser. Fiz. Tkh. Nauk. 3 (1959), 30-35.

[51] Yamada Y., Special polynomials and generalized Painlevé equations, in: Combinatorial Methods in Representation Theory, K. Koike, M. Kashiwara, S. Okada, I. Terada and H. F. Yamada (eds.), Adv. Stud. Pure Math. 28, Kinokuniya, Tokyo, Japan, (2000) 391-400.

[52] Zhedanov A. S., "Hidden symmetry" of Askey-Wilson polynomials, Theoret. and Math. Phys., 89 (1991) 1146-1157. 